ARTICLE

DOI: $10.1038 / s 41467-018-03847-z$

\title{
Release of Staphylococcus aureus extracellular vesicles and their application as a vaccine platform
}

Xiaogang Wang ${ }^{1}$, Christopher D. Thompson ${ }^{1}$, Christopher Weidenmaier (1) ${ }^{2,3}$ \& Jean C. Lee ${ }^{1}$

Secretion of extracellular vesicles (EVs), a process common to eukaryotes, archae, and bacteria, represents a secretory pathway that allows cell-free intercellular communication. Microbial EVs package diverse proteins and influence the host-pathogen interaction, but the mechanisms underlying EV production in Gram-positive bacteria are poorly understood. Here we show that EVs purified from community-associated methicillin-resistant Staphylococcus aureus package cytosolic, surface, and secreted proteins, including cytolysins. Staphylococcal alpha-type phenol-soluble modulins promote EV biogenesis by disrupting the cytoplasmic membrane; whereas, peptidoglycan cross-linking and autolysin activity modulate EV production by altering the permeability of the cell wall. We demonstrate that EVs purified from a $S$. aureus mutant that is genetically engineered to express detoxified cytolysins are immunogenic in mice, elicit cytolysin-neutralizing antibodies, and protect the animals in a lethal sepsis model. Our study reveals mechanisms underlying S. aureus EV production and highlights the usefulness of $E V s$ as a $S$. aureus vaccine platform.

\footnotetext{
${ }^{1}$ Division of Infectious Diseases, Department of Medicine, Brigham and Women's Hospital and Harvard Medical School, 181 Longwood Avenue, Boston, MA 02115, USA. ${ }^{2}$ Interfaculty Institute for Microbiology and Infection Medicine, University of Tuebingen, Elfriede Aulhorn Strasse 6, 72076 Tuebingen, Germany. ${ }^{3}$ German Center for Infection Research, Partner Site Tuebingen, 72076 Tuebingen, Germany. Correspondence and requests for materials should be addressed to J.C.L. (email: jclee@bwh.harvard.edu)
} 
taphylococcus aureus is a pathogenic bacterium that causes a wide spectrum of human infections, ranging from mild skin lesions to invasive, life-threatening infections. The pathogenesis of $S$. aureus infections is attributed to a wide array of virulence determinants, including surface proteins ${ }^{1}$ and glycopolymers ${ }^{2}$, as well as multiple secreted proteins, such as superantigens, leukotoxins, hemolysins, and proteases ${ }^{3}$. Although several specific export pathways have been described in S. aureus, the secretome often includes proteins that lack export signals and have typical cytoplasmic functions. The mechanisms by which cytoplasmic proteins are excreted by $S$. aureus have attracted recent interest ${ }^{4,5}$, and there is increasing evidence that these proteins may be secreted within extracellular membrane vesicles $(\mathrm{EVs})^{6-9}$.

EVs are nano-sized, spherical, bilayered membrane vesicles with a cargo that includes diverse proteins, polysaccharides, nucleic acids, and lipids. EV formation by Gram-negative bacteria was first observed by electron microscopy in the $1960 \mathrm{~s}^{10}$, and these bacteria secrete what are now referred to as outer membrane vesicles (OMVs). The generation of OMVs occurs by phospholipid accumulation in the outer leaflet of the outer membrane, followed by the formation of outer membrane protrusions that pinch off to form vesicles ${ }^{11}$. OMVs likely play important roles in bacterial pathogenesis due to packaging of multiple virulence factors ${ }^{12}$, and the ability of OMVs to serve as immune modulators by inducing innate and adaptive immune responses ${ }^{13}$.

Recent work has described the production and release of EVs from Gram-positive bacteria and fungi ${ }^{6-9,14}$. Because of the thick peptidoglycan (PGN) structure typical of Gram-positive microbes, EV biogenesis is a complex and poorly understood process. Toyofuku et al. ${ }^{14}$ recently reported that membrane vesicle formation in Bacillus subtilis was a result of prophageencoded endolysins that generated holes in the PGN, facilitating EV release. EVs from Gram-positive organisms play important roles in host-pathogen interactions, as supported by reports that EVs contain biologically active toxins, exhibit cytotoxicity, and elicit proinflammatory mediators ${ }^{9}$. Moreover, toxin-positive S. aureus EVs elicit skin barrier disruption in mice with characteristic atopic dermatitis-like skin inflammation ${ }^{15,16}$. The toxicity of staphylococcal EVs has hampered a relevant study of their immunogenicity and potential use as a vaccine platform.

Despite repeated efforts to develop experimental vaccines and immunotherapeutics against $S$. aureus, neither have proven effective in preventing staphylococcal infections in humans ${ }^{17}$. Mice immunized with native $S$. aureus EVs produced a robust T-cell response and were protected against lung infection, but EV toxicity was not addressed in this study ${ }^{18}$. The development of EVs as a $S$. aureus vaccine platforms will require characterization of the mechanisms of EV biogenesis to enable consistent production with adequate quality assurance.

In this study, we generate EVs from a predominant community-acquired, methicillin-resistant S. aureus (CA-MRSA) clone in the United States. Our study reveals distinct mechanisms that facilitate EV production at multiple stages. Phenol-soluble modulins (PSMs) act at the membrane level to facilitate vesicle budding at the cytoplasmic membrane; whereas, cell wall porosity is modulated by PGN cross-linking and production of autolysins. Our results demonstrate the cytotoxicity of native $S$. aureus EVs for multiple cell types. By genetically engineering a non-toxic $S$. aureus mutant to over-produce detoxified cytolysins, we show that engineered EVs (eng-EVs) are immunogenic, non-toxic, and

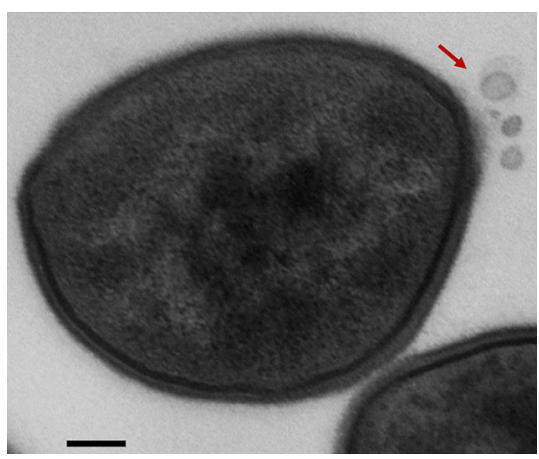

C

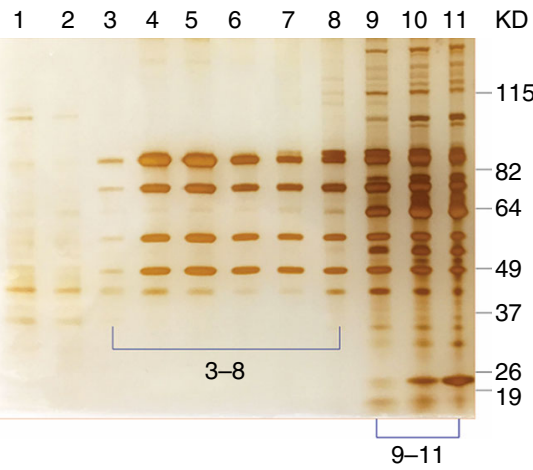

b

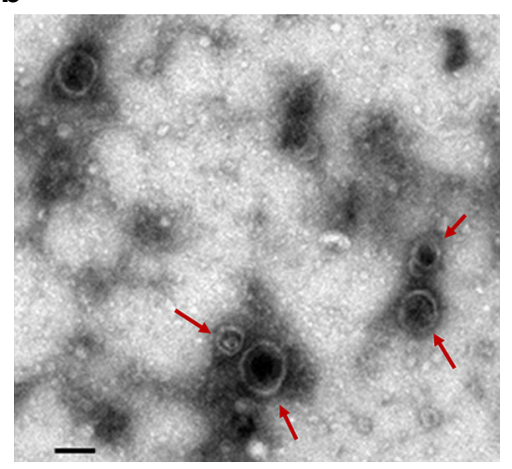

d

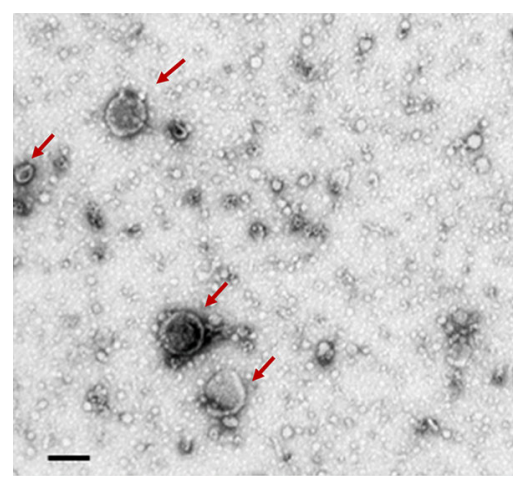

Fig. 1 Extracellular vesicles from S aureus JE2. The bacteria were cultivated in TSB. a Ultrathin sections of S. aureus JE2 examined by TEM revealed EVS (indicated by a red arrow) released from the cell wall. b Crude EVs (red arrows) pelleted by ultracentrigation from the JE2 culture supernatant were imaged by TEM. c EVs were purified by density gradient ultracentrifugation (Optiprep), and fractions were visualized by silver-stained SDS-PAGE. $\mathbf{d}$ Fractions 3-8 were pooled; OptiPrep was removed by diafiltration, and the samples were imaged by TEM. EVs were not visualized in fractions $9-11$. Scale bar, $100 \mathrm{~nm}$ 


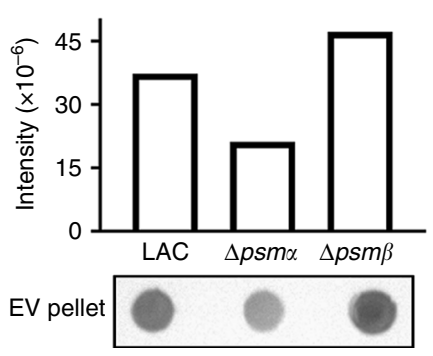

d

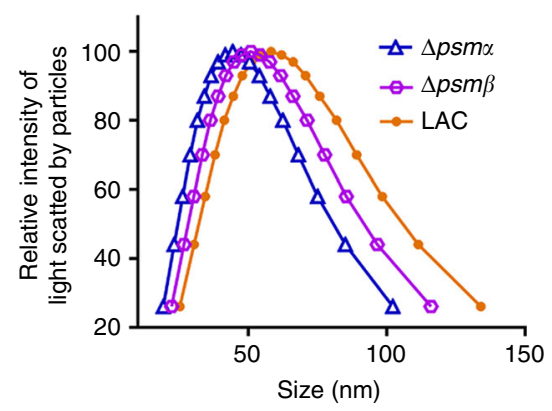

e

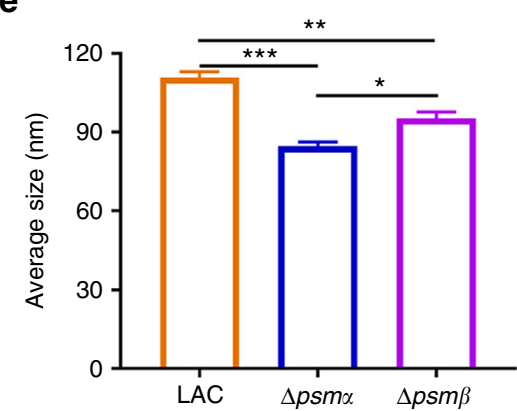

b

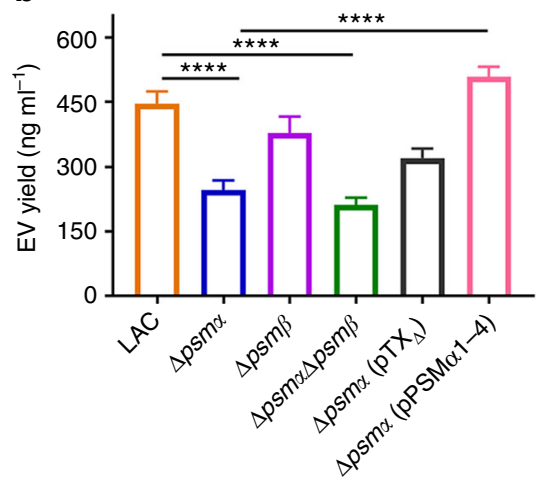

f

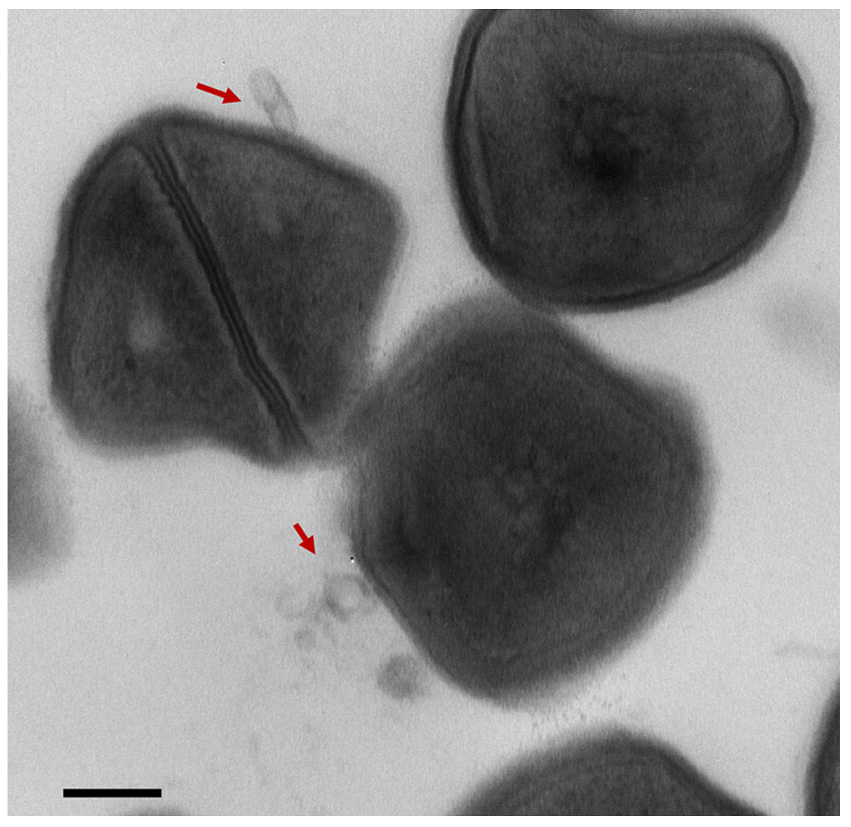

Fig. 2 Alpha-type phenol-soluble modulin peptides promote S. aureus EV production. a EV production from strain LAC and its isogenic mutants lacking psm $\alpha$ or $p \sin \beta$ was evaluated by dot-blotting EV suspensions purified from the same volume of bacterial culture, $\mathbf{b}$ by quantification of total EV protein abundance, or $\mathbf{c}$ by direct EV quantification of EV particles with nanoparticle tracking analysis. $\mathbf{d}$ The size distribution and $\mathbf{e}$ average size of EVs purified from WT and $\Delta p s m \alpha$ and $\Delta p s m \beta$ mutants were analyzed by dynamic light scattering, and the data were generated with ZataPALS particle sizing software. Dot-blotting was repeated at least twice, and a representitative result is presented. Signal intensity quantified by Image Studio Lite software is shown above the blot. EV quantification by other methods was calculated from at least three independent experiments and expressed as mean \pm s.e.m. $\mathbf{f}$ An electron micrograph of WT strain JE2 carrying $\mathrm{PTX}_{\Delta}$ expressing the genes encoding PSM $\alpha 1-4$ is shown. Scale bar, $200 \mathrm{~nm}$. Samples were compared with a Oneway ANOVA with Dunnett's multiple comparison test (Fig. 2b, c) or with Tukey's multiple comparison test (Fig. 2 e). ${ }^{\star} P<0.05$, ${ }^{\star \star} P<0.01,{ }^{\star \star \star} P<0.001$, $\star \star \star \star P<0.0001$

protect mice against $S$. aureus lethal sepsis. Our investigation describes a vaccine platform and provides the basis for further studies on the impact of EVs on the pathogenicity of $S$. aureus and other Gram-positive pathogens.

\section{Results}

Isolation of $S$. aureus EVs. Ultrathin sections of JE2 ${ }^{19}$ cells examined by transmission electron microscopy (TEM) revealed vesicle-like structures released from the $S$. aureus cell surface (Fig. 1a). We isolated the EVs by concentrating the culture supernatants to remove molecules $<100 \mathrm{kDa}$ before ultracentrifugation to pellet the EVs, shown in Fig. 1b. To remove non-membranous proteins, protein aggregates, and denatured EVs, Optiprep-based density gradient centrifugation was performed on the crude EV preparations. Consecutive Optiprep fractions $(10 \mu \mathrm{l})$ were subjected to SDS-PAGE. Little silverstained material was recovered from fractions 1 and 2 (Fig. 1c). Samples with similar protein banding patterns (fractions 3-8 and 9-11) were pooled, diafiltered, and examined by TEM. EVs were observed in fractions 3-8 (Fig. 1d), but not from fractions 9-11, indicating that EVs were distributed in fractions containing 20 $-35 \%$ Optiprep.

Protein composition of $S$. aureus EVs. A proteomic analysis of purified JE2 EVs by liquid chromatography-tandem mass spectrometry (LC-MS/MS) identified 165 proteins (Supplementary Data 1), including alpha toxin (Hla), leukocidin subunits (LukS-PV, LukF-PV, LukE, LukD, HlgB, and HlgC), adhesins (ClfA, ClfB, $\mathrm{SdrD}, \mathrm{SdrE}, \mathrm{Efb}$, and Ebh), MntC, proteases, and immune evasion factors (Sbi, phenol-soluble modulins, catalase, CHIPS, and SodA). 
a

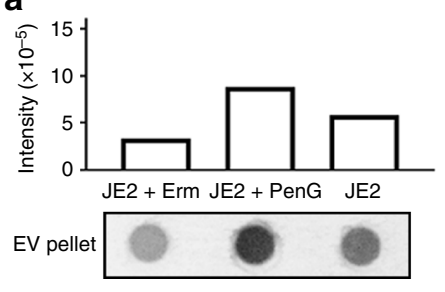

C

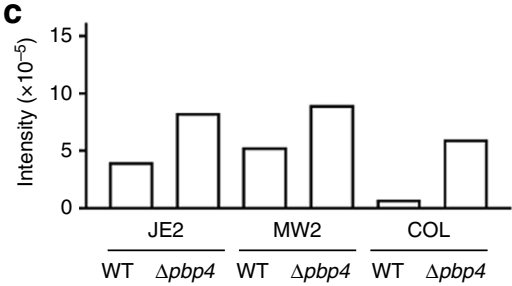

EV pellet $O P$
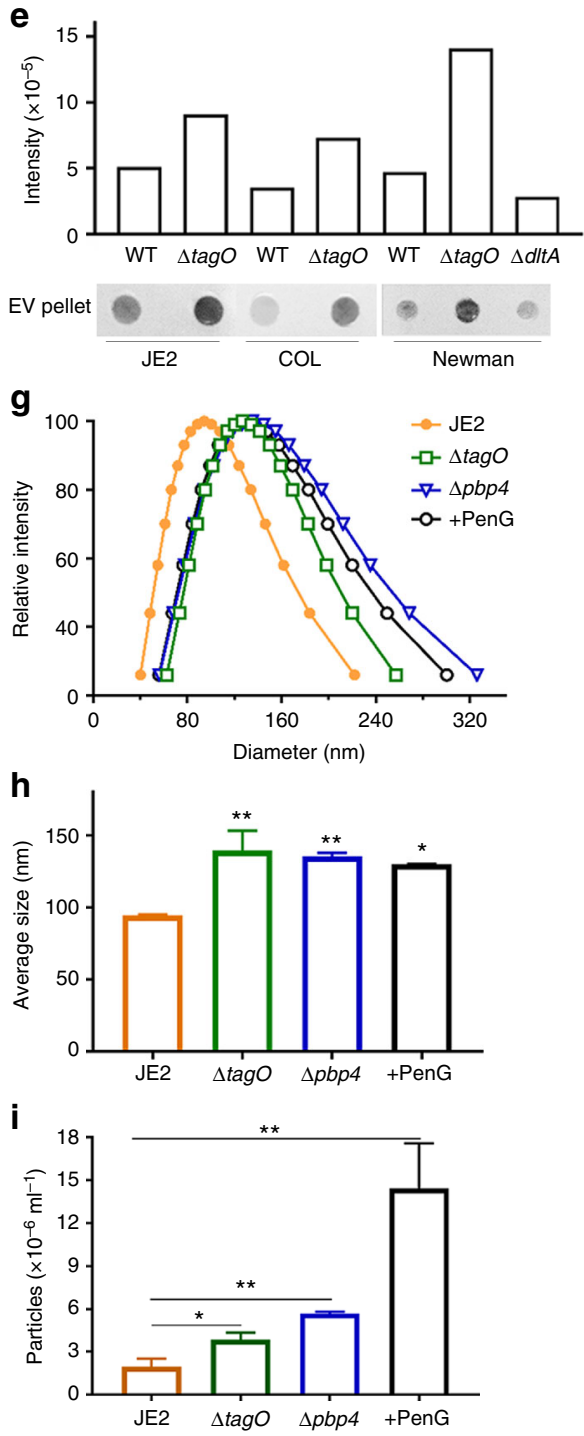

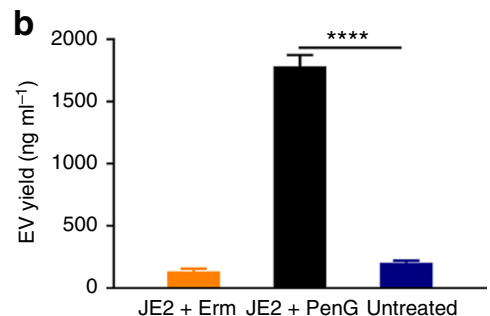

d

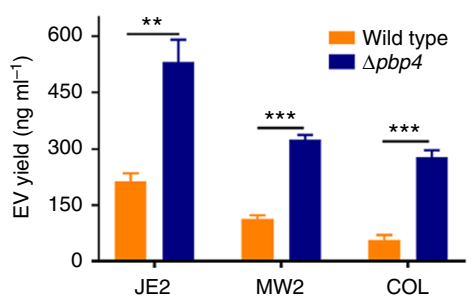

$\mathbf{f}$

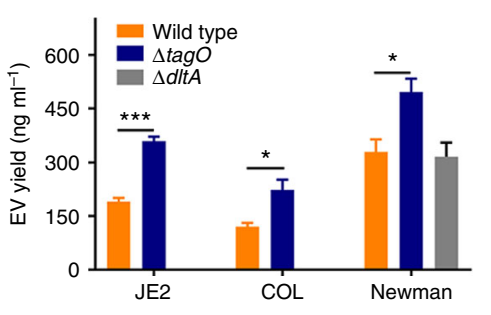

j

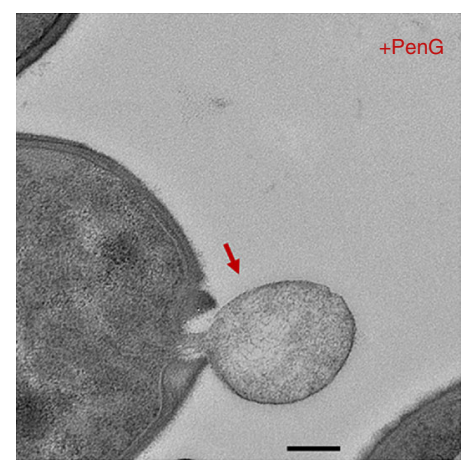

k

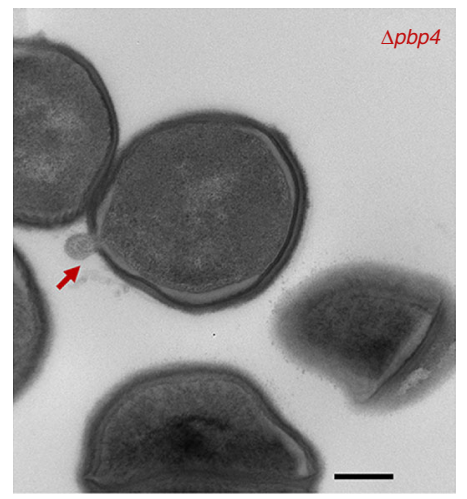


Other proteins of interest included penicillin-binding proteins, autolysins (Atl, Sle1, and other putative autolysins with predicted $\mathrm{N}$-acetylmuramoyl-L-alanine amidase activity), proteins involved in iron acquisition, and multiple other lipoproteins. Bioinformatic analyses revealed that $46 \%$ of $\mathrm{EV}$ proteins were cytoplasmic $(n=76), 16 \%$ were extracellular proteins $(n=27), 16 \%$ had an unknown localization $(n=27), 12 \%$ were membrane proteins $(n=19)$, and $10 \%$ were cell wall associated proteins $(n=16)$.

Phenol-soluble modulins promote EV release. S. aureus secretes PSMs, which are a family of amphipathic, alpha-helical, surfactant-like peptides that are proinflammatory and show cytolytic activity against neutrophils, erythrocytes, epithelial cells, and endothelial cells ${ }^{20,21}$. Alpha-type PSMs are required for mobilizing lipoproteins from the staphylococcal cytoplasmic membrane, a process essential for activating TLR2 ${ }^{22}$, as well as the export of cytoplasmic proteins, consistent with the membrane-damaging activity of PSMs ${ }^{5}$. Because the cargo of $S$. aureus EVs is enriched for both lipoproteins and cytoplasmic proteins, we evaluated whether PSM peptides were critical for EV generation.

We measured EV production by the WT USA300 LAC strain (the parent strain of JE2), as well as LAC $\Delta p s m \alpha, \Delta p s m \beta$, and $\Delta p \sin \alpha p \operatorname{si} \beta$ mutants $^{21}$. Dot immunoblot analysis revealed that deletion of psma genes reduced EV production (Fig. 2a). Likewise, protein assays and nanoparticle tracking analysis (NTA) indicated that the $p s m \alpha$ mutant showed significantly reduced $S$. aureus EV yield (Fig. 2b) and particle number (Fig. 2c), respectively. The $\Delta p s m \alpha$ and $\Delta p s m \alpha \Delta p s m \beta$ double mutant produced comparable levels of EVs (Fig. 2b), indicating that PSMa peptides play the dominant role in this phenotype. Complemention with $\mathrm{pTX}_{\Delta}$ expressing PSM $\alpha 1-4$ genes, but not the $\mathrm{pTX}_{\Delta}$ vector alone, restored EV production to the $\Delta p s m \alpha$ mutant (Fig. 2b, c). Mutation of the psm $\alpha$ genes significantly reduced $S$. aureus $\mathrm{EV}$ size (Fig. $2 \mathrm{c}, \mathrm{d}$ ); whereas, the $\Delta p \operatorname{si} \beta$ mutant produced EVs of intermediate size compared to that of wild-type (WT) LAC. We transduced $\mathrm{pTX}_{\Delta} \mathrm{PSM} \alpha 1-4^{21}$ into strain JE2, and its EV yield (protein content) increased from $184 \pm 12$ to $650 \pm$ $17 \mathrm{ng} \mathrm{ml}^{-1}(n=3)$. Nonetheless, electron micrographs of JE2 $\left(\mathrm{pTX}_{\Delta} \mathrm{PSM} \mathrm{1} 1-4\right)$ showed intact bacterial cells producing abundant EVs (Fig. 2f). Significant differences in bacterial numbers recovered from JE2 cultures with or without $\mathrm{pTX}_{\Delta} \mathrm{PSM} \alpha 1-4$ were not observed (Supplementary Fig. 1a), indicating minimal impact of $\mathrm{EV}$ formation on bacterial viability.

PGN cross-linking modulates EVs production. Unlike OMVs produced by Gram-negative microbes, $S$. aureus cytoplasmic membrane-derived EVs must traverse a PGN cell wall structure before cellular release. To determine whether the degree of PGN cross-linking affected $S$. aureus EV biogenesis, we cultured $S$. aureus JE2 in medium with a sublethal concentration $\left(0.2 \mu \mathrm{g} \mathrm{ml}^{-1}\right)$ of penicillin $G$ (PenG) that has been shown to decrease PGN crosslinking ${ }^{23}$. Treatment with a sublethal concentration $\left(0.1 \mu \mathrm{g} \mathrm{ml}^{-1}\right)$ of erythromycin (Em) served as an antibiotic control that has no effect on PGN cross-linking. Compared to EVs recovered from untreated cultures or cultures incubated with Em, the EV yield from PenG-treated cultures was distinctly higher (Fig. 3a). When the EV protein content was quantified from a fixed volume of culture left untreated or treated with sublethal antibiotic concentrations, we observed a 10-fold increase in EV yield from PenG-treated cultures (Fig. 3b). EV production had little effect on bacterial viability since differences were not observed in bacterial numbers recovered from JE2 cultured with or without PenG (Supplementary Fig. 1b).

S. aureus penicillin-binding protein 4 (PBP4) is a carboxypeptidase that is essential for secondary cross-linking of PGN, and a $p b p 4$ mutant shows a significant reduction in PGN crosslinking ${ }^{24}$. As predicted, both dot blot (Fig. 3c) and EV protein yield assays (Fig. 3d) showed increased EV production by JE2 $\Delta p b p 4$, and the protein yield was threefold higher than the wild-type JE2 strain. We also measured EV production in MRSA isolates MW2, COL, and their $\triangle p b p 4$ mutants; the relative increase in EV yield in the mutant strains (Fig. 3c, d) was consistent with that of JE2 $\Delta p b p 4$.

WTA is a PGN-anchored glycopolymer that is major component of the $S$. aureus cell wall and plays a critical role in cell wall homeostasis ${ }^{2}$. The $\operatorname{tagO}$ gene encodes an $\mathrm{N}$-acetyl glucosamine-phosphate transferase enzyme that catalyzes the first step in WTA biosynthesis ${ }^{25,26}$, and deletion of tagO gene abrogates $S$. aureus WTA production ${ }^{27}$. Compared to the WT strains JE2, COL, and Newman, tagO mutants showed an enhanced signal in the dot immunoblot assay for EV production (Fig. 3e). Likewise, quantitative analysis of EV protein yield showed that all three $\operatorname{tag} O$ mutants produced significantly more EVs than the parental isolates (Fig. 3f). Thus, WTAs negatively modulate $S$. aureus EV production, consistent with reports showing that $\operatorname{tagO}$ mutants are characterized by diminished PGN cross-linking ${ }^{28}$. The WTA backbone is decorated with esterlinked D-ala residues, which confer a zwitterionic charge to the polymer ${ }^{29}$. As shown in panels $\mathbf{e}$ and $\mathbf{f}$ of Fig. 3, production and yield of EVs by the $\Delta$ dltA mutant were similar to that of the parental strain Newman.

To determine whether EV size was affected by reduced PGN cross-linking, the size distribution of purified EVs was measured by dynamic light scattering (DSL). Treatment of JE2 cultures with PenG or mutation of $p b p 4$ or tagO resulted in a significant increase in the size distribution of EVs (Fig. 3g), as well as an increased EV average size (Fig. 3h) compared to untreated WT EVs. Because enhanced EV production and yield associated with reduced PGN cross-linking might be a result of larger EVs that would carry an increased cargo load, we quantified EVs by nanoparticle tracking analysis. As shown in Fig. 3i, treatment of JE2 cultures with PenG or mutation of $p b p 4$ or $\operatorname{tagO}$ resulted in suspensions containing significantly greater numbers of $\mathrm{EV}$

Fig. 3 Reductions in peptidoglycan cross-linking increase S. aureus EV production and size. a Dot blots were performed on JE2 EVs treated with subinhibitory concentrations of penicillin $\mathrm{G}$ (PenG) or erythromycin (Em) and probed with mouse EV antiserum. $\mathbf{b}$ EV protein abundance was quantified and expressed as ng EV protein $\mathrm{ml}^{-1}$ culture. c EV production from S. aureus strains JE2, MW2, COL, or their isogenic penicillin-binding protein 4 (pbp4) mutants was evaluated by dot-blotting EV suspensions or $\mathbf{d}$ by quantification of total EV protein yield. e EV production from strains JE2, COL, Newman, and their $\Delta \operatorname{tag} O$ (WTA-deficient) or $\triangle d$ ltA (lacking alanine modifications on WTA) mutants was evaluated by dot-blotting EV suspensions or $\mathbf{f}$ by quantification of total protein yield. $\mathbf{g}$ The size distribution and $\mathbf{h}$ average size of EVs isolated from JE2, PenG treated JE2, and $\Delta p b p 4$ and $\Delta$ tagO mutants were measured by dynamic light scattering. i EV particles from JE2, PenG treated JE2, and $\Delta p b p 4$ and $\Delta$ tagO mutants were quantified by nanoparticle tracking analysis. Electron micrographs of $\mathbf{j}$ JE2 cells treated with PenG or $\mathbf{k}$ carrying a pbp4 mutation are shown. Scale bar, $200 \mathrm{~nm}$. Dot immunoblot assays were performed at least twice with similar results; a representative blot is shown. EV protein yield and EV particle quantification experiments were calculated from at least three independent experiments and expressed as mean \pm s.e.m. The data were analyzed using one-way ANOVA with Dunnett's multiple comparison test (Fig. 3b, h, i) or using Student's t-test (Fig. 3d, f). ${ }^{\star} P<0.05,{ }^{\star \star} P<0.01,{ }^{\star \star \star} P<0.001,{ }^{\star \star \star \star} P<0.0001$ 
a

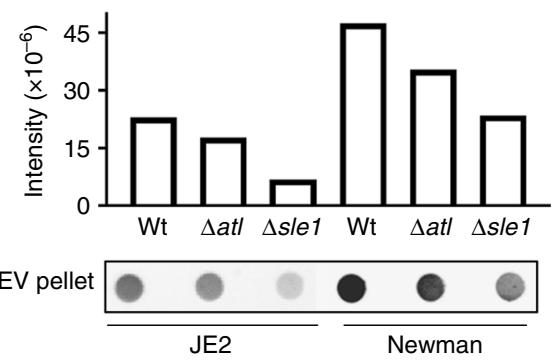

d

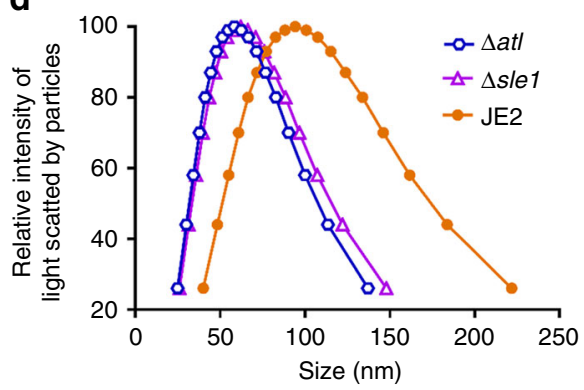

b

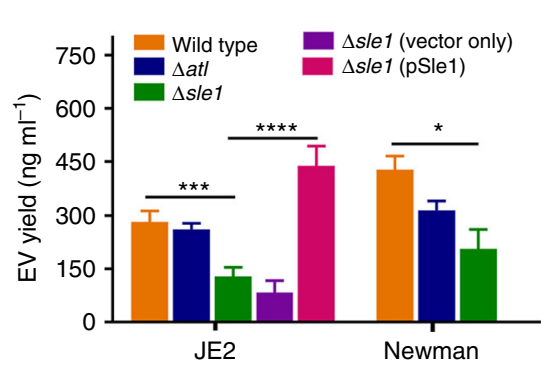

e

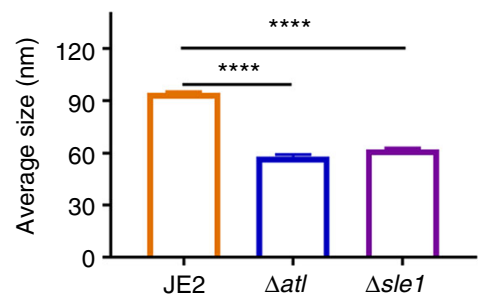

C

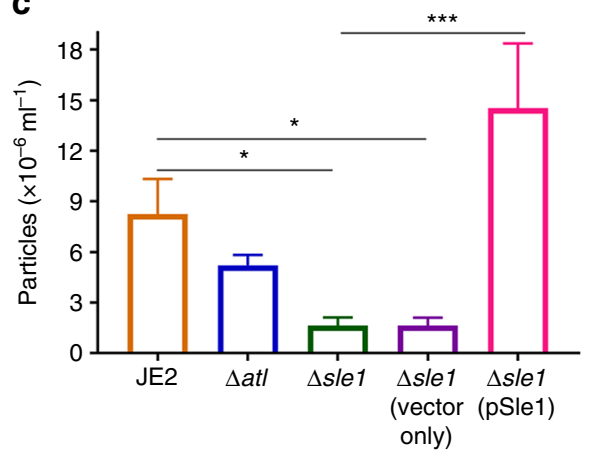

Fig. 4 The autolysin Sle1 promotes EV biogenesis. a EV production from JE2 and different autolysis mutants ( $\Delta$ atl and $\Delta$ sle1) was evaluated by dot-blotting EV suspensions, $\mathbf{b}$ by quantification of total EV protein abundance, or $\mathbf{c}$ by EV quantification using nanoparticle tracking analysis. $\mathbf{d}$ The size distribution and e average size of EVs isolated from JE2 and the $\Delta$ atl and $\Delta$ sle1 mutants were measured by dynamic light scattering. The dot immunoblot assay was repeated at least twice with similar results; a representative blot is shown. EV protein yield and EV particle quantification experiments were calculated from at least three independent experiments and expressed as mean \pm s.e.m. The data were analyzed using one-way ANOVA with Dunnett's multiple comparison test (Fig. 4b, c, e), For all panels, ${ }^{\star} P<0.05,{ }^{\star \star} P<0.01,{ }^{\star \star \star} P<0.001,{ }^{\star \star \star \star} P<0.0001$

particles per ml compared to untreated WT EVs. Electron micrographs of bacterial cells treated with PenG (Fig. 3j) or carrying a pbp4 mutation (Fig. 3k) showed EVs being released or budding, respectively, from the cell membrane. Taken together, our data indicate that $S$. aureus $\mathrm{EV}$ production is inversely proportional to the degree of PGN cross-linking.

Autolysin Sle1 promotes the release of EVs. Atl and Sle1 belong to a family of PGN hydrolases that plays a critical role in separation of daughter cells ${ }^{30,31}$, and Atl modulates the excretion of a subset of staphylococcal cytoplasmic proteins ${ }^{4}$. To determine whether PGN-hydrolases facilitate the release of EVs by altering the thick Gram-positive cell wall, we compared EV production from isogenic atl and sle1 mutants with that of strains JE2 and Newman. Although both mutants showed reduced EV production (Fig. 4a), the reduction in yield was only significant in the sle1 mutants (Fig. 4b). Likewise, NTA revealed that only the sle1 mutant yielded a significantly lower EV concentration compared to WT JE2 (Fig. 4c). Complemention with pSle1 expressing the sle1 gene, but not the pOS1-hprK vector alone, fully restored EV production to the JE2 $\Delta$ sle1 mutant (Fig. $4 \mathrm{~b}, \mathrm{c}$ ). Both atl and sle1 mutants exhibited significantly reduced EV size compared to WT JE2 EVs (Fig. 4d, e).

Bacteriophages also produce PGN hydrolases called endolysins, which degrade the bacterial cell wall from within, resulting in cell lysis and release of progeny phages. Recently, Toyofuku et al. ${ }^{14}$ reported that prophage-encoded endolysins mediate the formation and release of EVs from Bacillus subtilis by generating a hole in the cell wall, leading to cell death. To investigate whether prophages or prophage-encoded endolysins are involved in $S$. aureus EV production, we analyzed $S$. aureus strains NCTC 8325 carrying $\phi 11, \phi 12$, and $\phi 13$ and 8325-4, which is cured of all three prophages $^{32}$. Plating culture filtrates of NCTC 8325 on lawns of recipient strain $\mathrm{RN} 4220$ resulted in the formation of plaques, whereas culture filtrates of $8325-4$ yielded no plaques
(Supplementary Fig. 2a). EV yields and NTA revealed that NCTC 8325 and prophage-free strain $8325-4$ produced comparable level of EVs (Supplementary Fig. 2b and 2c), indicating that prophage mobilization is not essential for the generation of $S$. aureus EVs.

Effects of the $S$. aureus capsule on EV release. To determine whether the presence of capsular polysaccharide $(\mathrm{CP})$ production impacted $S$. aureus EV biogenesis, we evaluated a number of isogenic $\mathrm{CP}+$ and $\mathrm{CP}-$ strains. As shown in Fig. 5a, the $\mathrm{CP}$ phenotype had no obvious impact on the EV dot blot signal derived from WT or $\mathrm{CP}-$ mutants of strains Newman $(\mathrm{CP} 5+)$ or $6850(\mathrm{CP} 8+)$. Similarly, USA300 strain 923 (complemented to restore $\mathrm{CP} 5$ production) produced $\mathrm{CP} 5^{33}$, but there was no effect on the EV signal levels achieved by dot blotting (Fig. 5a). Likewise, $\mathrm{CP}+$ and isogenic $\mathrm{CP}$ - strains of Newman, 6850, and 923 produced comparable protein yields of EVs (Fig. 5b), indicating that $\mathrm{CP}$ did not modulate $S$. aureus EV production.

To investigate whether CP antigens were associated with $S$. aureus EVs, we performed CP immunoblots on EVs prepared from strains Newman (CP5+), MN8 (CP8+), and USA300 FPR3757 (CP-). CP antibodies react with surface-associated CP on intact EVs, whereas intravesicular $\mathrm{CP}$ would only be detected in sonicated EV preparations. Figure 5c shows that CP5 was only detected in sonicated, but not intact Newman EVs; whereas, CP8 was detected in both intact and sonicated MN8 EVs. EVs from CP- FPR3757 were non-reactive. Thus, both CP5 and CP8 were associated with EVs produced by $\mathrm{CP}+S$. aureus, although only CP8 was surface exposed.

Detoxified EVs as a multicomponent vaccine platform. Multiple antigens were packaged within JE2 EVs, including lipoproteins, cytolytic toxins, surface proteins, and enzymes (Supplementary Data 1). If the toxicity of the EVs were 
a

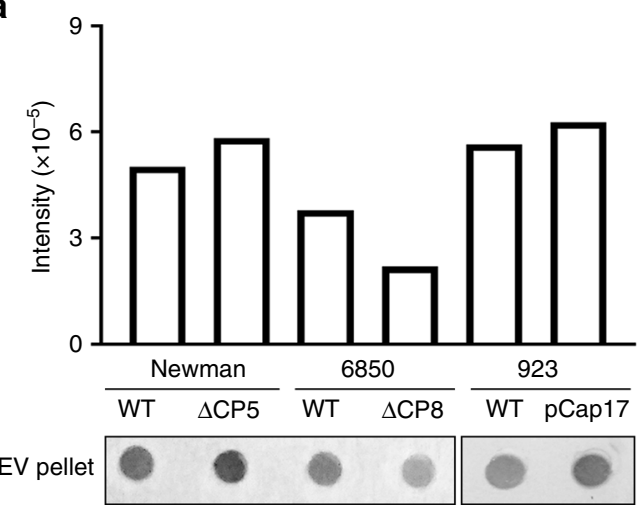

b

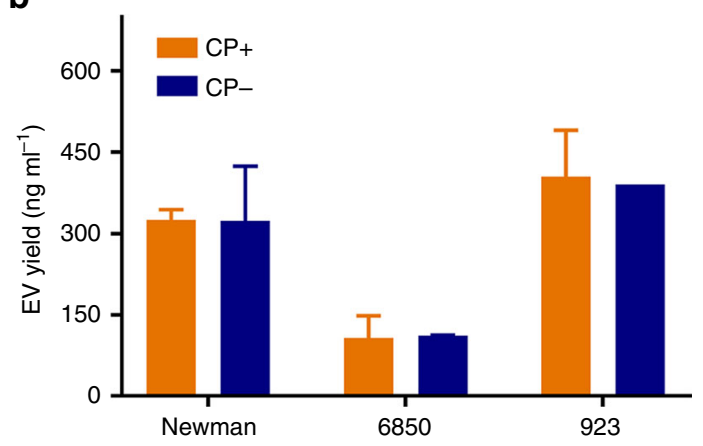

c

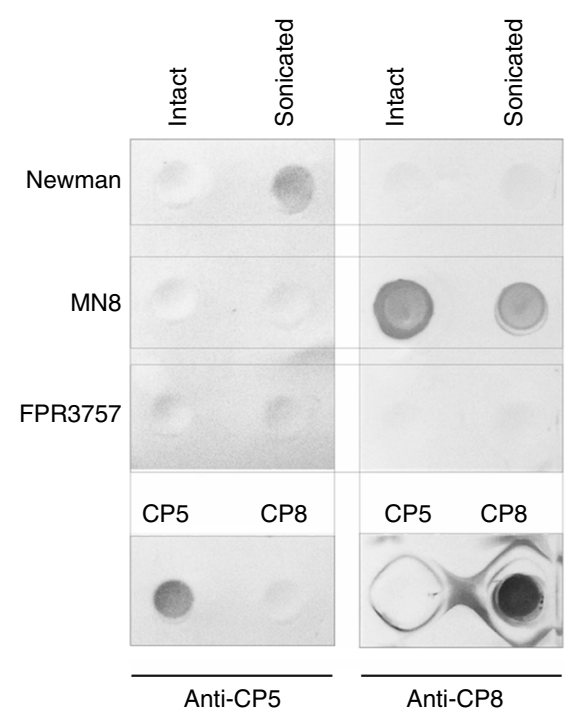

Fig. 5 Effects of S. aureus capsular polysaccharide synthesis on EV production. a EV production from encapsulated S. aureus (Newman, 6850, 923 [pCP5]) or their CP-negative counterparts (Newman $\Delta$ cap50, $6850 \Delta$ capHIJK, and 923) was evaluated by dot-blotting EV suspensions or b by quantification of total EV protein yield. c CP5 or CP8 was detected in intact or sonicated EVs $(35 \mu \mathrm{g})$ from strains Newman, MN8, or FPR3757 by immunoblots probed with $1.2 \mu \mathrm{g} \mathrm{m}^{-1} \mathrm{CP} 5$-specific $\mathrm{mAb} 4 \mathrm{C} 2$ or CP8-

specific mAb 5A6. Controls included $15 \mu \mathrm{g}$ purified CP5 and CP8. The dot immunoblot assay was repeated at least twice with similar results; a representative blot is shown. EV protein yields were calculated from at least three independent experiments and expressed as mean \pm s.e.m.

eliminated, JE2 EVs could serve as a multicomponent $S$. aureus vaccine candidate. To repress the expression of cytolytic toxins, we mutated the S. aureus global regulator agr in strain JE2. We subsequently deleted spa (the gene encoding protein A) since an agr mutant overexpresses Spa, which binds to the Fcy domain of immunoglobulin and dampens antibody development by crosslinking the Fab domain of $\mathrm{V}_{\mathrm{H}} 3$-type B-cell receptors ${ }^{34}$. The JE2 agr mutation significantly inhibited mRNA expression of hla (encoding alpha toxin) and the genes encoding all nine leukocidin subunits (Supplementary Fig. 3a). The JE2 $\Delta a g r \Delta s p a$ double mutant served as our $S$. aureus $\mathrm{EV}$ vaccine producing host strain. EVs from JE2, but not the JE2 $\Delta a g r \Delta s p a$ mutant, contained native Hla and LukE (Supplementary Fig. 3b) as assessed by western blotting. When we analyzed the protein content of JE2 $\Delta a g r \Delta s p a$ EVs by LC-MS/MS, many of the extracellular proteins present in JE2 WT EVs were not detected in JE2 $\Delta a g r \Delta s p a$ EVs. However, some antigens such as $\mathrm{MntC}$ and $\mathrm{FhuD} 2$ that protect mice against experimental S. aureus infections ${ }^{35,36}$ were present in EVs from the mutant strain. Neither protein A nor the toxins Hla, PantonValentine leukocidin (Luk-PVL), LukED, HlgCB, SelX, or PSMs were detectable in EVs purified from the JE2 $\Delta$ agr $\Delta$ spa mutant (Supplementary Data 2). Although LukAB was still present in EVs from JE2 $\Delta$ agr $\Delta$ spa, there was $\geq 86 \%$ reduction in the number of peptides detected in the mutant strain (Supplementary Data 1 and 2). Moreover, as indicated below, EVs recovered from the mutant strain showed no residual toxicity toward human leukocytes.

We immunized mice with $5 \mu \mathrm{g}$ EVs from JE2 $\Delta a g r$ or JE2 $\Delta$ agr $\triangle$ spa mutants; control mice were given phosphate buffered saline (PBS). EVs from both mutants elicited a serum antibody response against sonicated WT EVs, although the antibody level elicited by $\triangle a g r$ EVs was higher than that elicited by $\Delta a g r \Delta s p a$ EVs (Supplementary Fig. 4a). To examine the antigen profiles from EVs that elicited antibody responses after immunization, a bacterial lysate from USA300 strain FPR3757 was subjected to SDS-PAGE and immunoblotted with sera pooled from mice immunized with either $\Delta a g r$ EVs or $\Delta a g r \Delta s p a$ EVs. Sera from $\Delta a g r \Delta s p a$ EVs-immunized mice reacted with more bacterial antigens than sera from $\Delta a g r$ EVs-immunized mice (Supplementary Fig. $4 \mathrm{~b}$ ), suggesting that $\triangle a g r \Delta s p a$ EVs elicited a greater diversity of antibodies than $\Delta a g r$ EVs. The immunized mice were then challenged with strain FPR3757, a heterologous USA300 isolate. Immunization of mice with EVs from JE2 $\Delta$ agr $\Delta$ spa, but not EVs from JE2 $\triangle a g r$, provided significant protection against lethal sepsis (Supplementary Fig. 4c). Immunization with higher doses of JE2 $\Delta a g r \Delta s p a$ EVs mixed with alum did not significantly enhance immunogenicity (Supplementary Fig. 4d).

Engineered EVs protect mice against lethal sepsis. To enhance the protective efficacy of detoxified EVs from JE2 $\Delta a g r \Delta s p a$, we engineered JE2 to package non-toxic $\mathrm{Hla}_{\mathrm{H} 35 \mathrm{~L}}{ }^{37}$ and the LukE monomer within eng-EVs. LukED, detected in $82 \%$ of blood isolates and $61 \%$ of nasal isolates ${ }^{38}$, targets human and murine neutrophils, macrophages, T cells, dendritic cells, NK cells, and erythrocytes ${ }^{39}$.

We expressed non-toxic $\mathrm{Hla}_{\mathrm{H} 35 \mathrm{~L}}$ and LukE in strain JE2 $\Delta$ agr $\Delta$ spa under control of the spa promoter, which is enhanced in an $\Delta a g r$ genetic background ${ }^{40}$. Thus, mRNA levels of $\mathrm{Hla}_{\mathrm{H} 35 \mathrm{~L}}$ and LukE expressed in JE2 $\Delta a g r \Delta s p a$ were dramatically increased compared to expression in JE2 $\Delta a g r \Delta$ spa or JE2 $\Delta a g r \Delta$ spa with the empty vector (Supplementary Fig. $3 \mathrm{c}$ ). Both $\mathrm{Hla}_{\mathrm{H} 35 \mathrm{~L}}$ and LukE were detected by western blot in eng-EVs isolated from recombinant strain JE2 $\Delta a g r \Delta s p a\left(\mathrm{pHla}_{\mathrm{H} 35 \mathrm{~L}}\right.$-LukE) (Supplementary Fig. 3b).

The relative toxicity of EVs prepared from WT strain JE2 and JE2 $\Delta a g r \Delta s p a$ vs. eng-EVs from JE2 $\Delta a g r \Delta s p a\left(\mathrm{pHla}_{\mathrm{H} 35 \mathrm{~L}}\right.$-LukE) was assessed by incubating EVs in vitro with three different cell types. A549 cells are susceptible to Hla-mediated cytolysis, and WT strain JE2 EVs were toxic for A549 cells at concentrations as low as $1 \mu \mathrm{g} \mathrm{ml}^{-1}$. In contrast, JE2 $\Delta$ agr $\Delta$ spa mutant EVs and the 
a

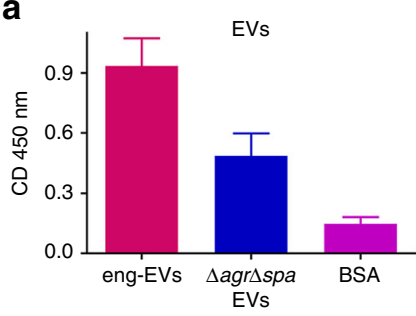

e

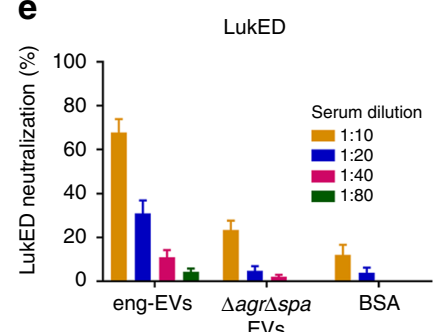

b

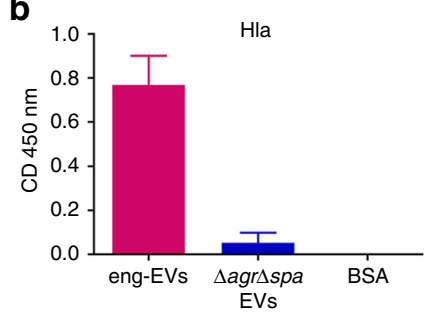

$\mathbf{f}$

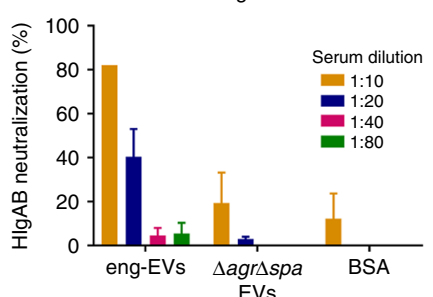

C

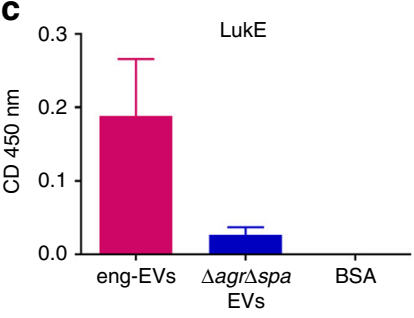

g

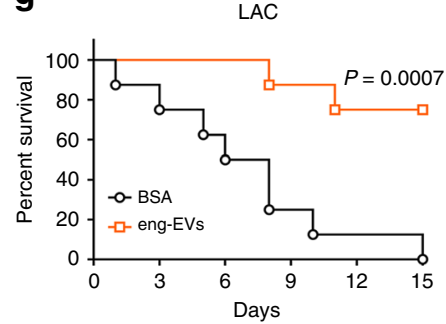

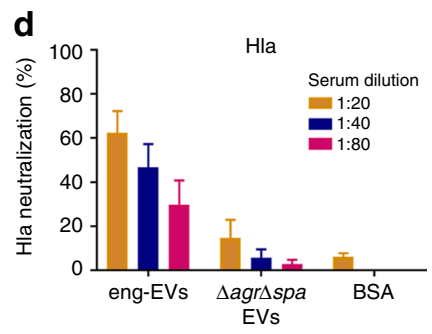

h

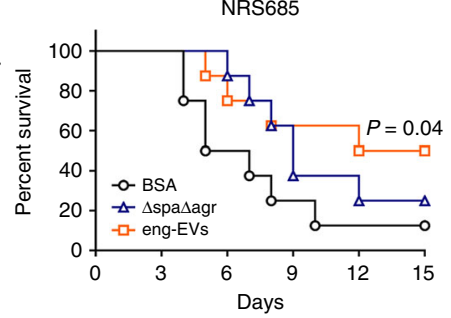

Fig. 6 Immunogenicity and protective efficacy in mice of engineered-EVs. Antibody levels in sera (diluted 1:100) from mice immunized with eng-EVs were analyzed on ELISA plates coated with a JE2 sonicated EVs, b Hla, or c LukE. Data were expressed as mean \pm s.e.m. The neutralizing activity of sera from mice immunized with BSA or different EV preparations was determined by either incubating serial dilutions of sera with $\mathbf{d} H \mathrm{Hla}$, e LukED, or $\mathbf{f}$, or HIgAB for $1 \mathrm{~h}$ at $37^{\circ} \mathrm{C}$ before adding target cells. Control cells were incubated with toxins but no sera. Data are expressed as percent neutralization $\pm \mathrm{s}$.e.m. Mice $(n=8)$ immunized with different JE2 EV preparations were challenged IV with $8 \times 10^{7}$ CFU S. aureus LAC (g) or $2 \times 10^{8}$ NRS685 (h). Survival (comparing EV-immunized mice vs. BSA-immunized mice) was analyzed with the log-rank test

eng-EVs from JE2 2 agr $\Delta s p a\left(\mathrm{pHla}_{\mathrm{H} 35 \mathrm{~L}}\right.$-LukE) exhibited negligible toxicity (Supplementary Fig. 5a). HL60 cells are susceptible to the cytolysis induced by $S$. aureus leukocidins, and JE2 EVs, but not $\Delta a g r \Delta s p a$ or eng-EVs, were cytolytic for HL60 cells (Supplementary Fig. 5b). Rabbit erythrocytes are susceptible to Hla, PSMs, and the leukocidins HIgAB and LukED ${ }^{39}$. JE2 EVs exhibited significant hemolytic activity, whereas no hemolysis resulted from $\Delta a g r \Delta s p a$ mutant EVs or eng-EVs (Supplementary Fig. 5c). These data demonstrate that the eng-EVs were non-toxic in vitro for mammalian cells.

We immunized mice on days 0,14 , and 28 with $5 \mu$ g eng-EVs from JE2 $\Delta a g r \Delta s p a\left(\mathrm{pHla}_{\mathrm{H} 35 \mathrm{~L}}\right.$-LukE) or with $5 \mu \mathrm{g}$ EVs from the JE2 $\Delta a g r \Delta s p a$ mutant; control mice received $5 \mu \mathrm{g}$ bovine serum albumin (BSA). Whereas sera from mice immunized with both eng-EVs and $\Delta a g r \Delta s p a$ EVs, but not BSA, reacted by ELISA with sonicated WT JE2 EVs (Fig. 6a), only mice given the eng-EVs responded with antibodies to purified Hla (Fig. 6b) or LukE (Fig. 6c). These data indicate that recombinant proteins packaged within $S$. aureus $\mathrm{EV}$ are immunogenic.

To examine whether the antibodies elicited by the eng-EV vaccine were functional, toxin neutralizing assays were performed. Sera from mice immunized with eng-EVs effectively neutralized Hla at dilutions ranging from 1:20 to 1:80 (Fig. 6d), whereas serum neutralizing antibodies were low or undetectable in the BSA or $\Delta a g r \Delta s p a$ EV groups. Similarly, sera from mice immunized with eng-EVs, but not BSA or $\Delta a g r \Delta s p a \mathrm{EVs}$, were able to effectively neutralize LukED at dilutions ranging from 1:10 to 1:20 (Fig. 6e). Sera from mice immunized with eng-EVs also neutralized leukocidin HlgAB (Fig. 6f), but not PVL-SF or HlgCB leukotoxins.

The immunized mice were challenged with USA300 strain LAC or USA500 strain NRS685, a PVL-negative MRSA bacteremia isolate. We chose the latter strain because the PVL$S$ and PVL-F subunits can interact with LukE and LukD to form inactive hybrid complexes, which have been shown to influence LukED-mediated $S$. aureus virulence in mice ${ }^{41}$. As shown in Fig. 6g, h, immunization with eng-EVs provided significant protection against both $S$. aureus isolates in the lethal murine sepsis model. JE2 $\Delta a g r \Delta s p a$ EVs were not protective against the USA500 strain (Fig. 6h).

\section{Discussion}

The production of membrane vesicles represents a secretory pathway common to mammalian cells, fungi, and bacteria that allows cell-free intercellular communication ${ }^{42-44}$. Microbial EVs encapsulate cargo that include lipids, proteins, glycans, and nucleic acids, which have been shown to play roles in microbial physiology, pathogenesis, and the transmission of biological signals into host cells to modulate biological processes and host innate immune responses ${ }^{42,43,45,46}$. In Gram-negative bacteria, EVs are generated by pinching off the outer membrane, but the mechanism(s) by which EVs escape the thick cell walls of Gram-positive bacteria, mycobacteria, and fungi are unknown. Once shed, S. aureus EVs can undergo cholesterol-dependent fusion with host cell membranes to deliver their toxic cargo ${ }^{47}$. S. aureus EVs are produced in vivo during experimental pneumonia in mice ${ }^{47}$. In this report, we demonstrate unique properties associated with $\mathrm{EV}$ production by JE2, a $S$. aureus USA300 strain representative of the prevalent CA-MRSA clone in the US. Similar to EVs characterized from other $S$. aureus isolates ${ }^{47-49}$, JE2 EVs encapsulate an array of bacterial antigens, including lipoproteins, exotoxins, and cytoplasmic proteins.

In this report, we evaluated putative factors that modulate the membrane and PGN related steps of EV release. PSMs are a group of small alpha helical peptides that have surfactant-like properties and potent cytolytic activity for leukocytes, epithelial cells, and endothelial cells ${ }^{20}$. PSMa peptides are 20-22 amino acids in length; whereas, PSM $\beta$ peptides are 43-45 amino acids in length. In our studies, PSM $\alpha$ peptides, but not PSM $\beta$ peptides, supported the generation of EVs from S. aureus. EVs from the PSMa mutant were less abundant and smaller in size compared with WT EVs. Chatterjee et al. ${ }^{50}$ reported that an $S$. aureus mutant that lacks the PSM transporter protein accumulates PSMs intracellularly, causing cytoplasmic membrane perturbations. 
Surfactants or surfactant-like proteins with amphipathic helical structures have been shown to insert into lipid monolayers and generate local deformation ${ }^{51,52}$. PSMs, due to their surfactant-like activity, as well as amphipathic helical structure, may enhance membrane curvature under cytoplasmic turgor pressure, resulting in membrane disruption and the formation of EVs. Although EVs from Gram-negative bacteria arise from the outer membrane rather than the plasma membrane, the biogenesis of OMV production is also thought to be due to perturbations in the outer leaflet of the membrane due to specific phospholipid accumulation therein ${ }^{11}$. Recently, Ebner et al. ${ }^{5}$ reported that $S$. aureus PSMa peptides-induced the cellular release of cytoplasmic proteins, lipids, nucleic acids, and ATP into culture supernatants, and that this effect was mediated by the membrane-damaging activity of the PSMa peptides. Because PSMa peptides promote EV production, and EVs encapsulate cytoplasmic proteins, lipids, and nucleic acids ${ }^{18}$ within a bilayered membrane, we postulate that these released cellular components are associated with and are likely contained within EVs.

The S. aureus cell envelope is comprised of a thick, highly cross-linked PGN layer, proteins, and glycopolymers like lipoteichoic acid, WTA, and CP. Highly cross-linked PGN serves as a barrier for EV biogenesis since treatment of $S$. aureus with a sublethal concentration of PenG or genetic inactivation of $p b p 4$ or tagO resulted in a significant increase in $\mathrm{EV}$ production and size. This inverse correlation between PGN cross-linking and EV yield was also observed with $S$. aureus strains MW2, COL, and Newman. WTA has been shown to be critical for PGN-cross-linking by regulating PBP4 localization to the septation site ${ }^{28}$. A secondary mechanism by which WTA regulates EV production is via its ability to control the activity of Atl and Sle1-not only by preventing their binding to $S$. aureus cell wall $\mathrm{PGN}^{53,54}$, but also by creating an acidic milieu that limits Atl PGN hydrolase activity $^{55}$. Consequently, autolytic activity is not localized to the septum area in a tagO mutant but is spread throughout the cell surface, likely facilitating EV release. Schlag et al. ${ }^{53}$ reported that a $\operatorname{tag} O$ mutant showed an altered cell surface with bobble- and hairy-like protrusions, which may represent EVs. Although we do not yet fully understand the mechanism(s) of EV generation in Gram-positive bacteria, it seems logical that a poorly cross-linked cell wall or a cell wall lacking WTA would lessen the barrier to EV release and generate larger EVs as a result of larger pores within the PGN structure.

Autolysins that cleave the PGN barrier also impact the biogenesis of $S$. aureus EVs. Atl and Sle1 localize to the septum during cell division where they exhibit peptidoglycan hydrolase activity, resulting in separation of the daughter cells ${ }^{53,54}$. Sle1 is a $32 \mathrm{kDa}$ protein comprised of an $\mathrm{N}$ terminal cell wall binding domain and a $\mathrm{C}$ terminal catalytic domain with $\mathrm{N}$-acetyl muramyl-L-alanine amidase activity. In contrast, Atl is a $138 \mathrm{kDa}$ bifunctional PGN hydrolase that is processed to yield a $62 \mathrm{kDa}$ protein with amidase activity (similar to that of Sle1) and a 51 $\mathrm{kDa}$ protein with endo- $\beta-\mathrm{N}$-acetyl glucosaminidase activity. Atl is also involved in cell wall turnover and penicillin- or detergentinduced bacterial autolysis. Deletion of sle1, but not atl, significantly reduced $S$. aureus EV production. Pasztor et al. ${ }^{4}$ reported that an SA113 atl mutant overexpressed eight putative secondary PGN hydrolases both at the transcriptional and at the protein levels, highlighting the supplementary role of these alternative autolysins in the absence of Atl. This observation may at least partially explain why JE2 $\Delta a t l$ and Newman $\Delta a t l$ showed only a modest reduction in EV yield. Mutation of atl only slightly reduced $\mathrm{EV}$ yield, but the average size of EVs from the mutant was smaller than that of WT JE2, suggesting that Atl is involved in EV biogenesis. Atl modulates the excretion of staphylococcal cytoplasmic proteins ${ }^{4}$, and it is likely that $\mathrm{EV}$ production at least partially explains the Atl-mediated shedding of cytoplasmic proteins in S. aureus.

Although both autolysin activities are localized to the $S$. aureus septum region, JE2 EVs are not confined to the septal region (Fig. 2f), and EVs have been visualized by others surrounding the bacterial surface $7,48,56$. A recent report demonstrated differential roles for Atl and Sle1 during cell division and separation ${ }^{57}$. Whereas Sle1 could be visualized over the entire septal surface, Atl localized only at the external (surface exposed) edge of the septum $^{58}$. How autolysins modulate EV release from the cell wall or whether this process is spatially or temporally regulated remains to be determined.

We reported that $S$. aureus CP was shed from broth-grown $S$. aureus cells ${ }^{59}$, and it is feasible that EVs could serve as a vehicle to liberate CP from the cell envelope. The Streptococcus pneumoniae capsule was reported to hinder EV release in this pathogen ${ }^{7}$, whereas no effect was observed on EV yield in strains with or without the hyaluronic capsule of Streptococcus pyogenes ${ }^{8}$. Whether these streptococcal CPs are present as EV cargo in these pathogens was not addressed. Although EV yield varied among different isolates, we recovered similar quantities of EVs from isogenic $S$. aureus strains that varied only in CP production. The glucuronoxylomannan capsule of Cryptococcus neoformans has been identified as a component of EVs from this fungal pathogen $^{60}$, and polysaccharide A from Bacteroides fragilis was shown to be packaged into OMVs that were capable of inducing immunomodulatory signaling in dendritic cells ${ }^{46}$. Ongoing studies in our laboratory will address whether S. aureus EV-host cell interactions impact the pathogenesis of staphylococcal disease.

We considered that $S$. aureus EVs could serve as a vaccine platform if their cytotoxicity was abrogated, and this was accomplished by purifying EVs from an $\Delta a g r \Delta s p a$ mutant of strain JE2. To enhance the protective efficacy of the $\Delta a g r \Delta s p a \mathrm{EV}$ vaccine, non-toxic $\mathrm{Hla}_{\mathrm{H} 35 \mathrm{~L}}$ and LukE were expressed in JE2 $\Delta a g r \Delta s p a$ under the control of the agr-derepressed spa promoter. Immunization with purified non-toxic $\mathrm{Hla}_{\mathrm{H} 35 \mathrm{~L}}$ has been shown to prevent lethal pneumonia and lethal peritonitis and reduce the incidence of necrotic skin abscesses ${ }^{61-63}$. S. aureus leukocidins comprise a family of pore-forming cytolysins produced by $S$. aureus that target monocytes, lymphocytes, neutrophils, and macrophages - the very cells responsible for resolution of bacterial infection. These "eng-EVs" elicited antibodies in the sera of immunized mice that reacted with Hla and LukE by ELISA and neutralized the cytolytic activity of Hla, LukED, and $\mathrm{Hlg} A B$ in vitro.

Immunization with eng-EVs provided significant protection against lethal sepsis provoked by USA300 strain LAC, a virulent PVL+ isolate. Because of a report that the presence of PVL modulates LukED-mediated $S$. aureus virulence in mice ${ }^{41}$, we challenged another group of immunized mice with USA500 strain NRS685, a PVL-negative MRSA bacteremia isolate. Immunization with eng-EVs, but not $\triangle a g r \Delta s p a$ EVs, protected $50 \%$ of the mice against NRS685 lethal sepsis. Protective efficacy against additional $S$. aureus strains and in additional infection models remains to be evaluated. Overexpression of additional antigens that have been shown to protect mice against experimental $S$. aureus infections, such as $\mathrm{MntC}$ and FhuD2 $2^{35,36}$, in secondgeneration eng-EVs may yield a more broadly protective vaccine. LC-MS/MS analysis of EVs from both WT JE2 and the $\Delta a g r \Delta s p a$ mutant strain contained multiple lipoproteins. As a predominant TLR2 ligand, lipoproteins have been increasingly used as adjuvant components ${ }^{64,65}$ because they are potent activators of host innate immunity and can mediate humoral and cell mediated immune responses. The self-adjuvanting composition of eng-EVs may provide it with a unique advantage over purified component vaccines. 


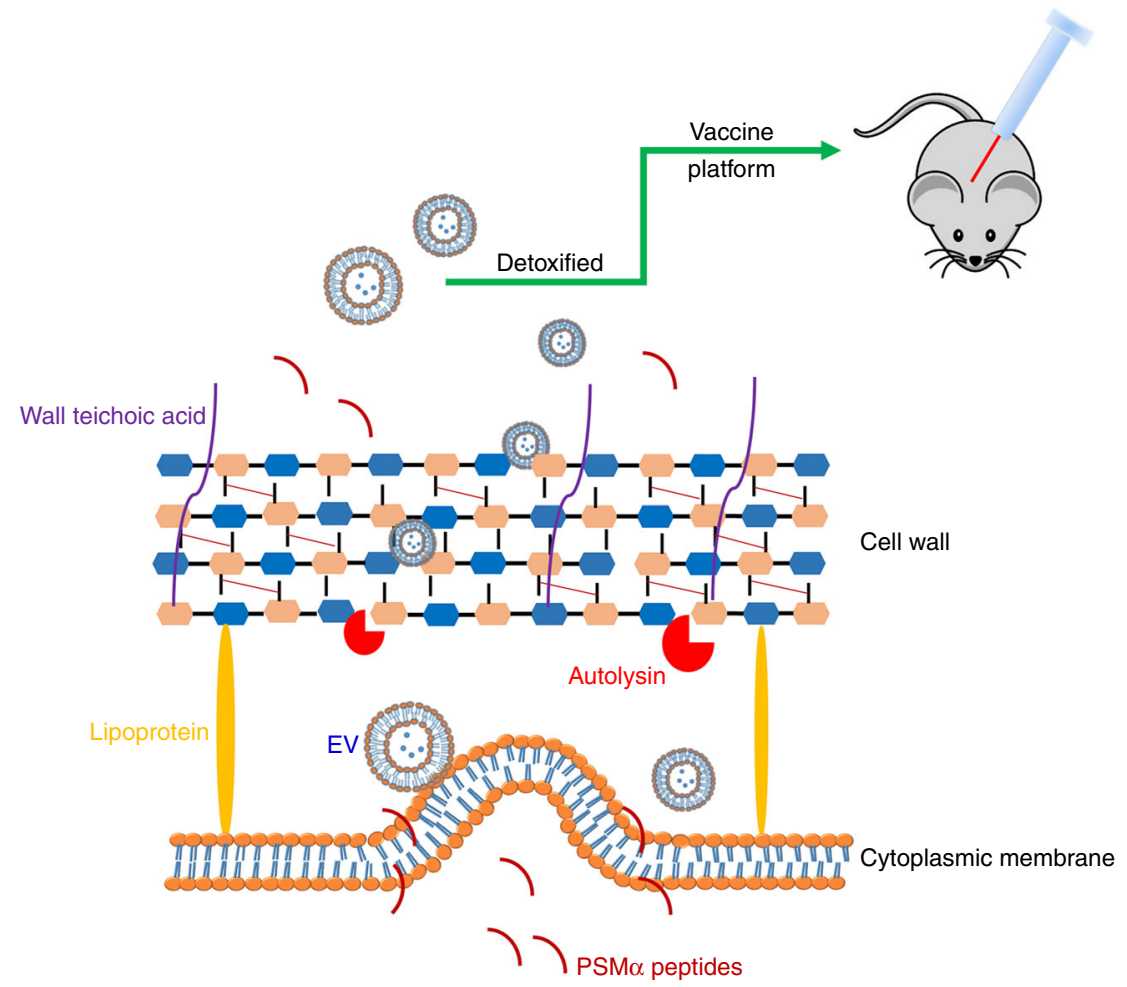

Fig. 7 Proposed mechanisms underlying EV production by Staphylococcus aureus. EVs are generated from the cytoplasmic membrane, and this process is promoted by S. aureus PSM $\alpha$ peptides, which have surfactant-like activity, causing membrane disruption. Membrane-derived EVs must also traverse the highly cross-linked S. aureus peptidoglycan barrier, and the extent of cell wall cross-linking modulates the efficiency of EV production. Autolysins, such as Sle1, facilitate EV release by hydrolyzing peptidoglycan, particularly at sites of active cell division. We mutated S. aureus to render its EVs non-toxic, and then genetically engineered the mutants to package detoxified antigens in EVs. These recombinant EVs were immunogenic in mice and showed protective efficacy in a sepsis model of $S$. aureus infection

In summary, we have generated, purified, and characterized EVs isolated from $S$. aureus USA300, the predominant CA-MRSA clone in the United States. Our study revealed that $S$. aureus PSMs are central for EVs generation by targeting the cytoplasmic membrane. Likewise, the Sle1 autolysin was shown to be critical for the release of EVs from the S. aureus cell wall. Whereas mutations in Atl or CP production did not affect EV yield, PBP4 and WTA promoted PGN cross-linking and consequently diminished EV production. Our study elucidates certain mechanisms whereby $S$. aureus produces and sheds EVs (Fig. 7) and will ultimately further our understanding of bacterial physiology and pathogenesis. We designed and created eng-EVs as a vaccine platform against $S$. aureus infection. Detoxified EVs that over-produced Hla $\mathrm{H}_{35 \mathrm{~L}}$ and LukE were immunogenic, elicited toxin neutralizing antibodies, and protected mice in a $S$. aureus lethal sepsis model, indicating that these naturally produced vesicles have potential as a noval vaccine platform.

\section{Methods}

Bacterial strains and plasmids. S. aureus isolates (listed in Supplementary Table 1) were cultivated overnight with aeration in tryptic soy broth (TSB; Difco) at $37^{\circ} \mathrm{C}$. Escherichia coli strain XL-10 (Agilent), used in DNA cloning experiments, was grown at $37^{\circ} \mathrm{C}$ in Luria Broth (LB; Difco). S. aureus-E. coli shuttle vector pCU $1^{66}$ was used for cloning and expression of appropriate genes in S. aureus, and pOS1- $h p r K$ was used for pSle1 complementation studies. Antibiotics were added in the following concentrations: PenG $0.2 \mu \mathrm{g} \mathrm{ml}^{-1}$, ampicillin (Amp; $100 \mu \mathrm{g} \mathrm{ml}^{-1}$ ), Em $5 \mu \mathrm{g} \mathrm{ml}^{-1}$, chloramphenicol $\left(\mathrm{Cm} ; 10 \mu \mathrm{g} \mathrm{ml}^{-1}\right.$ ), kanamycin (Kan; $50 \mu \mathrm{g} \mathrm{ml}^{-1}$ ), and tetracycline (Tet; $5 \mu \mathrm{g} \mathrm{ml}^{-1}$ ).

DNA manipulation. Fey et al. ${ }^{19}$ derived S. aureus JE2 from the USA300 strain LAC by curing it of plasmids, rendering it sensitive to Em. The agr mutation ( $\triangle$ agr:: tet $M$ ) was transduced from S. aureus RN6911 ${ }^{40}$ to WT JE2 using bacteriophage $\phi 80 a$ with selection for Tet resistance. To construct the JE2 $\triangle a g r \Delta s p a$ double mutant, the spa mutation was transduced from JE2 (spa::ermB) to JE2 $\triangle a g r$ by $\phi 80 a$ transduction. The $p b p 4$ mutation was transduced from JE2 $(\Delta p b p 4:: e r m B)$ to WT MW2 and COL by $\phi 80 \alpha$ transduction with selection for Em resistance. All mutants were confirmed by PCR using the primers listed in Supplementary Table 2. ELISA results confirmed the phenotype of the $\triangle s p a$ mutant, and the agr mutant lost its hemolytic phenotype. To construct the WTA mutants, the tagO mutation was transduced from SA113 $\Delta$ tagO (pRBtagO) to WT JE2 and COL with $\phi 80 a$ with selection for Em resistance. Mutants were confirmed by PCR and acquisition of resistance to lysis by $\phi 80 \alpha$. To complement the JE2 $\Delta$ sle 1 mutant, a DNA fragment of $1005 \mathrm{bp}$ containing the sle1 gene was amplified from JE2 genomic DNA using the primer pair listed in Supplementary Table 2. The Sle1 expression plasmid was constructed by cloning the slel gene under the control of the $h p r K$ promoter into the E.coli-S. aureus shuttle vector pOS1 ${ }^{67}$. pSle1 was transformed into RN4220 by electroporation and then transduced to JE2 $\Delta$ sle 1 by $\phi 80 a$ transduction.

To construct a shuttle vector for expression of $\mathrm{Hla}_{\mathrm{H} 35 \mathrm{~L}}$ and LukE, the spa promoter, $h l a_{H 35 L}$, and lukE genes were amplified from S. aureus strains JE2, DU1090 $\left(\mathrm{pHla}_{\mathrm{H} 35 \mathrm{~L}}\right.$ ), and FPR3757, respectively, using the primers listed in Supplementary Table 2. To drive the expression of $h a_{H 35 L}$, its sequence was fused to the $3^{\prime}$ terminus of the spa promoter containing the ribosome binding site by overlapping PCR. The $\mathrm{P}_{s p a}-$ hla $a_{H 35 L}$ fusion sequence was cloned into the shuttle plasmid pCU1 with restriction enzymes HindIII and SalI. The amplified lukE sequence containing a ribosome binding site was inserted into pCU1 with restriction enzymes SalI and EcoRI. The resulting plasmid pCU1-P $\mathrm{P}_{s p a}-h l a_{H 35 L}-l u k E$ was verified by enzyme digestion and DNA sequencing. To construct JE2 $\Delta$ spa $\Delta a g r$ expressing non-toxic $\mathrm{Hla}_{\mathrm{H} 35 \mathrm{~L}}$ and LukE, pCU1- $\mathrm{P}_{s p a}-h \mathrm{hl}_{\mathrm{H} 35 \mathrm{~L}}-\mathrm{lukE}$ was transformed into RN4220 by electroporation and then transduced with $\phi 80 a$ to JE2 $\Delta s p a \Delta a g r$, selecting for $\mathrm{Cm}$ resistance.

Isolation and purification of EVs. S. aureus was cultivated in TSB with shaking to an $\mathrm{OD}_{650 \mathrm{~nm}}$ of 1.2 . The culture supernatant was filtered and concentrated 25 -fold with a $100-\mathrm{kDa}$ tangential flow filtration system (Pall Corp.). The retentate was filtered again before centrifugation at $150,000 \times g$ for $3 \mathrm{~h}$ at $4^{\circ} \mathrm{C}$ to pellet the vesicles and leave soluble proteins in the supernatant. The EV pellet was suspended in $40 \%$ Optiprep density gradient medium (Sigma) and overlaid with gradient layers of Optiprep ranging from 35 to $10 \%$. After centrifugation at $139,000 \times g$ for $16 \mathrm{~h}$ at $4^{\circ} \mathrm{C}, 1 \mathrm{ml}$ fractions were removed sequentially from the top of the gradient. Each fraction was subjected to SDS-PAGE and stained with a Thermo Fisher silver staining kit. Fractions with a similar protein profile on SDS-PAGE were pooled, 
and the Optiprep medium was removed by diafiltration with PBS using an Amicon Ultra-50 Centrifugal Filter Unit. The diafiltered retentate was filtered $(0.45 \mu \mathrm{m})$ and stored at $4{ }^{\circ} \mathrm{C}$. EV protein concentrations were determined by using a Protein Assay Dye Reagent (Bio-Rad). EV samples were evaluated with a Nanobrook ZetaPALS potential analyzer (Brookhaven Instruments Corp.), and the data for size distribution and particle diameter were generated with ZetaPALS particle sizing software. Nanoparticle tracking analysis was performed by purifying EVs from 100 $\mathrm{ml}$ bacterial cultures, as described above. The number of EV particles recovered from individual cultures (and suspended in $1 \mathrm{ml}$ PBS) was determined using a Nanosight NS300 Sub Micron Particle Imaging System (Malvern). A camera level of 12 and a gain of 1 was utilized to optimize data collection, and analyses were performed with Nanoparticle Tracking and Analysis software (NTA 3.1). Each sample was analyzed three times for $30 \mathrm{~s}$ at $20^{\circ} \mathrm{C}$ using different fields of view. Frame sequences were analyzed under manual particle detection and tracking parameters (screen gain of 4 and detection threshold of 17).

Transmission electron microscopy. For imaging S. aureus ultrathin sections, the cultures were fixed with $2.5 \%$ paraformaldehyde, $5 \%$ glutaraldehyde, and $0.06 \%$ picric acid in cacodylate buffer and postfixed with $1 \%$ osmium tetroxide/ $1.5 \%$ potassium ferrocyanide. After incubation in $1 \%$ uranyl acetate, samples were sequentially dehydrated in ethanol before they were soaked in propylene oxide and infiltrated overnight with propylene oxide and TAAB Epon. Ultrathin sections were stained with lead citrate. To image EVs, $5 \mu \mathrm{l}$ S. aureus EVs were adsorbed for $1 \mathrm{~min}$ to a carbon coated grid that was made hydrophilic by a 30-s exposure to a glow discharge. The samples were stained with $0.75 \%$ uranyl formate. All samples were examined in a TecnaiG2 Spirit BioTWIN transmission electron microscope, and images were recorded with an AMT $2 \mathrm{k}$ CCD camera.

Proteomic analysis of EVs by LC-MS/MS. S. aureus EVs ( $8-10 \mu \mathrm{g})$ were subjected to SDS-PAGE and stained with Coomassie Blue R-250. Gel sections were analyzed at the Taplin mass spectrometry facility at Harvard Medical School using an LTQ Orbitrap Velos Pro ion-trap mass spectrometer (Thermo Fisher). Peptide sequences (and hence protein identity) were determined by matching protein databases with the acquired fragmentation pattern using the software program Sequest (Thermo Fisher Scientific). Proteins were identified by a minimum of two peptides and at least one unique peptide. Sequence analysis was performed with a database containing protein sequences of the S. aureus USA300 FPR3757 genome downloaded from NCBIprot. The subcellular localization of each identified protein was predicted by PsortB v.3.0 (www.psort.org/psorb/).

Real-time RT-PCR assay. S. aureus strains were cultivated in $5 \mathrm{ml} \mathrm{TSB}$ at $37^{\circ} \mathrm{C}$ to an $\mathrm{OD}_{650 \mathrm{~nm}}$ of 0.9 . After centrifugation at $4{ }^{\circ} \mathrm{C}$, the bacterial cells were mixed with glass beads in $300 \mu$ lysis buffer (RNeasy mini kit; Qiagen) and lysed by using a high speed Ultramat 2 Amalgamator (SDI, Inc.). Total RNA from the lysate supernatant was purified with the RNeasy mini kit (Qiagen), treated with DNase I (Invitrogen), and stored at $-70^{\circ} \mathrm{C}$. cDNA was synthesized from $1 \mu \mathrm{g}$ of bacterial RNA using a Protoscript II First Strand cDNA synthesis kit (New England Biolabs). A total of $50 \mathrm{ng}$ of synthesized cDNA was subjected to Real-time RT-PCR using a Power Green PCR Master Mix (Applied Biosystems) with primers listed in Supplementary Table 2 and detected in a StepOnePlus Real-Time PCR System (Applied Biosystems). The relative transcriptional levels of $h l a_{H 35 L}$ and $l u k E$ were calculated using the $\Delta \Delta \mathrm{Ct}$ method by normalizing to the 16S rRNA transcriptional level.

Immunoblotting assays. For western blots, $10 \mu \mathrm{g}$ S. aureus EVs were subjected to SDS-PAGE, transferred to nitrocellulose membranes, and blocked with PBS + $0.05 \%$ Tween-20 (PBST) and $1 \%$ skim milk for $1 \mathrm{~h}$ at room temperature (RT). After washing with PBST, the membranes were incubated with rabbit anti-LukS-PV $\left(2 \mu \mathrm{g} \mathrm{m}^{-1}\right.$; IBT Bioservices; Cat. No. 04-0009) or mouse anti-Hla monoclonal antibody (mAb) $6 \mathrm{C} 12\left(1 \mu \mathrm{g} \mathrm{ml}^{-1}\right.$; IBT Bioservices; Cat. No. 0210-005) overnight at $4{ }^{\circ} \mathrm{C}$. The membranes were washed and incubated with HRP-conjugated goat antirabbit IgG (1:5000; ImmunoReagent, Inc.; Cat. No. GtxRb-004-DHRPX) or antimouse IgG (1:5000; ImmunoReagent, Inc.; Cat. No. GtxMu-004-DHRPX) for $2 \mathrm{~h}$ at RT before developing the blots using TMB membrane peroxidase substrate (Kirkegaard \& Perry Laboratories, Inc). Purified Hla (List Biological Labs) and LukE (IBT Bioservices) were used as positive controls. Uncropped scans of the western blots are shown in Supplementary Fig. 6.

For EV dot blotting assays, intact or sonicated EVs were applied to nitrocellulose membranes using a 96-well dot blotter system (Bio-Rad). The membranes were immersed in PBST $+5 \%$ skim milk for $2 \mathrm{~h}$ before blocking the staphylococcal IgG binding proteins (Spa and Sbi) by overnight incubation at $4{ }^{\circ} \mathrm{C}$ with an irrelevant human IgG1 monoclonal antibody $\left(10 \mu \mathrm{g} \mathrm{ml}^{-1}\right)$ in PBST $+1 \%$ skim milk. The membrane was washed with PBST and incubated overnight at $4{ }^{\circ} \mathrm{C}$ with sera (diluted 1:1000 in PBST $+1 \%$ skim milk) pooled from mice immunized with EVs (see below) or murine mAbs ${ }^{59}$ to CP5 $\left(4 \mathrm{C} 2 ; 1.2 \mu \mathrm{g} \mathrm{ml}^{-1}\right.$ ) or CP8 (5A6; 1.2 $\mu \mathrm{g} \mathrm{ml}^{-1}$ ). After washes with PBST, the membrane was incubated with alkaline phosphatase (AP)-conjugated goat anti-mouse antibody (1:15,000; Sigma; Cat. No. A2429) at RT for $2 \mathrm{~h}$. The membrane was washed with PBST and developed with AP membrane substrate (KPL).
EV cytotoxicity. The relative toxicity of $S$. aureus EVs $\left(1-20 \mu \mathrm{g} \mathrm{ml}^{-1}\right)$ toward human A549 lung epithelial cells (ATCC), neutrophil-like HL60 cells (ATCC), and rabbit erythrocytes (Hardy Diagnostics) was assessed. A549 lung epithelial cells grown in a 96-well plate were incubated overnight at $37^{\circ} \mathrm{C}$ with EVs or $1 \mu \mathrm{g} \mathrm{ml}^{-1}$ of purified Hla. Toxicity was assessed using an LDH cytotoxicity assay kit (Thermo Fisher Scientific). Differentiated HL60 cells $\left(2 \times 10^{5}\right.$ cells) were seeded in a 96-well plate and treated with EVs or $1 \mu \mathrm{g} \mathrm{ml} l^{-1}$ of PVL for $4 \mathrm{~h}$ at $37^{\circ} \mathrm{C}$. Cell viability was measured with a CellTiter kit (Promega). A $2 \%$ rabbit erythrocyte suspension was mixed with EVs or $1 \mu \mathrm{g} \mathrm{ml}^{-1} \mathrm{Hla}$ in a 96-well plate for $1 \mathrm{~h}$ at $37^{\circ} \mathrm{C}$. The erythrocytes were pelleted by centrifugation, and hemolysis was recorded by measuring the $\mathrm{OD}_{545 \mathrm{~nm}}$ of the supernatant using an ELISA reader.

Animal studies. Mouse experiments were carried out in accordance with the recommendations in the PHS Policy on Humane Care and Use of Laboratory Animals, and animal use protocols were approved by the Partners Healthcare Institutional Animal Care and Use Committee. Female Swiss Webster mice ( 4 weeks old; Charles River) were immunized by the subcutaneous route on days 0 , 14 , and 28 with $5 \mu \mathrm{g}$ of $\Delta a g r$ EVs, $\Delta a g r \Delta s p a$ EVs, or eng-EVs. Control animals were immunized similarly with BSA (Sigma). Blood was collected from the mice by tail vein puncture before each vaccination and again before challenge. Sera were diluted 1:100 and tested by ELISA on 96-well plates coated with $5 \mu \mathrm{g} \mathrm{ml}^{-1}$ sonicated WT EVs, $5 \mu \mathrm{g} \mathrm{ml}^{-1}$ LukE, or $1 \mu \mathrm{g} \mathrm{ml}^{-1}$ Hla. Immunized mice were inoculated with $0.8-2 \times 10^{8}$ CFU S. aureus by intravenous (IV) tail vein injection 2 weeks after the third vaccination. Survival was monitored up to 15 days post challenge, and the data were analyzed using the log-rank test.

Toxin neutralization assays. For the Hla neutralization assays, mouse serum samples were pre-incubated with native Hla for $1 \mathrm{~h}$ before the addition of $2 \%$ rabbit erythrocytes. After $1 \mathrm{~h}$, the cytotoxicity of the serum-neutralized samples was measured by recording the $\mathrm{OD}_{545 \mathrm{~nm}}$ of the sample supernatants ${ }^{68}$. For leukocidin neutralization assays, blood was collected from healthy volunteers giving written informed consent, as approved by the Institutional Review Board of The Brigham and Women's Hospital (Human Subject Assurance Number 00000484). Neutrophils were isolated from $10 \mathrm{ml}$ blood using Polymorphprep (Accurate Chemical), washed, and suspended in RPMI (Invitrogen) containing 5\% fetal bovine serum (Invitrogen). Sera from immunized mice were serially diluted and mixed with toxin concentrations yielding $\sim 75 \%$ cell lysis $\left(12.5 \mu \mathrm{g} \mathrm{ml} l^{-1}\right.$ LukED, $2.5 \mu \mathrm{g} \mathrm{ml}$ PVL, $1 \mu \mathrm{g} \mathrm{ml}^{-1} \mathrm{HlgAB}$, or $2 \mu \mathrm{g} \mathrm{ml}^{-1} \mathrm{HlgCB}$ (1:1 S and F subunits). Samples were pre-incubated with leukocidins for $30 \mathrm{~min}$ at RT before the addition of neutrophils $\left(1.2 \times 10^{5}\right.$ cells). After $2 \mathrm{~h}$ at $37^{\circ} \mathrm{C}$ in $5 \% \mathrm{CO}_{2}$, the cells were collected by centrifugation and suspended in fresh medium. Cell cytotoxicity was evaluated using a CellTiter kit (Promega). Percent neutralization was calculated using the formula: [\% cytotoxicity of (toxin + cells) $-\%$ cytotoxicity of (serum + toxin + cells)].

Data availability. Data supporting the findings of this manuscript are available from the corresponding author upon reasonable request. Mass spectrometry proteomics data were deposited in the ProteomeXchange Consortium (http://proteomecentral.proteomexchange.org) via the PRIDE partner repository with the data set identifier PXD007953.

Received: 27 October 2017 Accepted: 14 March 2018 Published online: 11 April 2018

\section{References}

1. Geoghegan, J. A. \& Foster, T. J. Cell wall-anchored surface proteins of Staphylococcus aureus: many proteins, multiple functions. Curr. Top. Microbiol. Immunol. 409, 95-120 (2017).

2. Weidenmaier, C. \& Lee, J. C. Structure and function of surface polysaccharides of Staphylococcus aureus. Curr. Top. Microbiol. Immunol. 409, 57-93 (2016)

3. Otto, M. Staphylococcus aureus toxins. Curr. Opin. Microbiol. 17, 32-37 (2014)

4. Pasztor, L. et al. Staphylococcal major autolysin (Atl) is involved in excretion of cytoplasmic proteins. J. Biol. Chem. 285, 36794-36803 (2010).

5. Ebner, P. et al. Non-classical protein excretion is boosted by PSMalphainduced cell leakage. Cell Rep. 20, 1278-1286 (2017)

6. Rivera, J. et al. Bacillus anthracis produces membrane-derived vesicles containing biologically active toxins. Proc. Natl Acad. Sci. USA 107, 19002-19007 (2010).

7. Olaya-Abril, A. et al. Characterization of protective extracellular membranederived vesicles produced by Streptococcus pneumoniae. J. Proteom. 106, $46-60$ (2014)

8. Resch, U. et al. A two-component regulatory system impacts extracellular membrane-derived vesicle production in group A Streptococcus. mBio 7, e00207-e00216 (2016) 
9. Brown, L., Wolf, J. M., Prados-Rosales, R. \& Casadevall, A. Through the wall: extracellular vesicles in Gram-positive bacteria, mycobacteria and fungi. Nat. Rev. Microbiol. 13, 620-630 (2015).

10. Knox, K. W., Vesk, M. \& Work, E. Relation between excreted lipopolysaccharide complexes and surface structures of a lysine-limited culture of Escherichia coli. J. Bacteriol. 92, 1206-1217 (1966).

11. Roier, S. et al. A novel mechanism for the biogenesis of outer membrane vesicles in Gram-negative bacteria. Nat. Commun. 7, 10515 (2016).

12. Ellis, T. N. \& Kuehn, M. J. Virulence and immunomodulatory roles of bacterial outer membrane vesicles. Microbiol. Mol. Biol. Rev. 74, 81-94 (2010).

13. Kim, J. H., Lee, J., Park, J. \& Gho, Y. S. Gram-negative and Gram-positive bacterial extracellular vesicles. Semin. Cell Dev. Biol. 40, 97-104 (2015).

14. Toyofuku, M. et al. Prophage-triggered membrane vesicle formation through peptidoglycan damage in Bacillus subtilis. Nat. Commun. 8, 481 (2017).

15. Hong, S. W. et al. An important role of alpha-hemolysin in extracellular vesicles on the development of atopic dermatitis induced by Staphylococcus aureus. PLoS ONE 9, e100499 (2014).

16. Jun, S. H. et al. Staphylococcus aureus-derived membrane vesicles exacerbate skin inflammation in atopic dermatitis. Clin. Exp. Allergy 47, 85-96 (2017).

17. Fowler, V. G. Jr \& Proctor, R. A. Where does a Staphylococcus aureus vaccine stand? Clin. Microbiol Infect. 20, 66-75 (2014). Suppl 5.

18. Choi, S. J. et al. Active immunization with extracellular vesicles derived from Staphylococcus aureus effectively protects against staphylococcal lung infections, mainly via Th1 cell-mediated immunity. PLoS ONE 10, e0136021 (2015).

19. Fey, P. D. et al. A genetic resource for rapid and comprehensive phenotype screening of nonessential Staphylococcus aureus genes. mBio 4, e00537-00512 (2013).

20. Cheung, G. Y., Joo, H. S., Chatterjee, S. S. \& Otto, M. Phenol-soluble modulins--critical determinants of staphylococcal virulence. FEMS Microbiol. Rev. 38, 698-719 (2014).

21. Wang, R. et al. Identification of novel cytolytic peptides as key virulence determinants for community-associated MRSA. Nat. Med. 13, 1510-1514 (2007).

22. Hanzelmann, D. et al. Toll-like receptor 2 activation depends on lipopeptide shedding by bacterial surfactants. Nat. Commun. 7, 12304 (2016)

23. Wyke, A. W., Ward, J. B., Hayes, M. V. \& Curtis, N. A. A role in vivo for penicillin-binding protein-4 of Staphylococcus aureus. Eur. J. Biochem. 119, 389-393 (1981).

24. Leski, T. A. \& Tomasz, A. Role of penicillin-binding protein 2 (PBP2) in the antibiotic susceptibility and cell wall cross-linking of Staphylococcus aureus: evidence for the cooperative functioning of PBP2, PBP4, and PBP2A.J. Bacteriol. 187, 1815-1824 (2005).

25. Xia, G. \& Peschel, A. Toward the pathway of S. aureus WTA biosynthesis. Chem. Biol. 15, 95-96 (2008).

26. Brown, S., Zhang, Y. H. \& Walker, S. A revised pathway proposed for Staphylococcus aureus wall teichoic acid biosynthesis based on in vitro reconstitution of the intracellular steps. Chem. Biol. 15, 12-21 (2008).

27. Weidenmaier, C. et al. Role of teichoic acids in Staphylococcus aureus nasal colonization, a major risk factor in nosocomial infections. Nat. Med. 10, 243-245 (2004).

28. Atilano, M. L. et al. Teichoic acids are temporal and spatial regulators of peptidoglycan cross-linking in Staphylococcus aureus. Proc. Natl Acad. Sci. USA 107, 18991-18996 (2010).

29. Weidenmaier, C., McLoughlin, R. M. \& Lee, J. C. The zwitterionic cell wall teichoic acid of Staphylococcus aureus provokes skin abscesses in mice by a novel CD4+T-cell-dependent mechanism. PLoS ONE 5, el3227 (2010).

30. Biswas, R. et al. Activity of the major staphylococcal autolysin Atl. FEMS Microbiol. Lett. 259, 260-268 (2006).

31. Kajimura, J. et al. Identification and molecular characterization of an Nacetylmuramyl-L-alanine amidase Sle1 involved in cell separation of Staphylococcus aureus. Mol. Microbiol. 58, 1087-1101 (2005).

32. Novick, R. Properties of a cryptic high-frequency transducing phage in Staphylococcus aureus. Virology 33, 155-166 (1967).

33. Boyle-Vavra, S. et al. USA300 and USA500 clonal lineages of Staphylococcus aureus do not produce a capsular polysaccharide due to conserved mutations in the cap5 locus. mBio 6, e02585-02514 (2015).

34. Falugi, F., Kim, H. K., Missiakas, D. M. \& Schneewind, O. Role of protein A in the evasion of host adaptive immune responses by Staphylococcus aureus. mBio 4, e00575-00513 (2013).

35. Mishra, R. P. et al. Staphylococcus aureus FhuD2 is involved in the early phase of staphylococcal dissemination and generates protective immunity in mice. $J$. Infect. Dis. 206, 1041-1049 (2012).

36. Anderson, A. S. et al. Staphylococcus aureus manganese transport protein C is a highly conserved cell surface protein that elicits protective immunity against S. aureus and Staphylococcus epidermidis. J. Infect. Dis. 205, 1688-1696 (2012).

37. Menzies, B. E. \& Kernodle, D. S. Passive immunization with antiserum to a nontoxic alpha-toxin mutant from Staphylococcus aureus is protective in a murine model. Infect. Immun. 64, 1839-1841 (1996).
38. von Eiff, C., Friedrich, A. W., Peters, G. \& Becker, K. Prevalence of genes encoding for members of the staphylococcal leukotoxin family among clinical isolates of Staphylococcus aureus. Diagn. Microbiol. Infect. Dis. 49, 157-162 (2004).

39. Reyes-Robles, T. \& Torres, V. J. Staphylococcus aureus pore-forming toxins. Curr. Top. Microbiol. Immunol. 409, 121-144 (2016).

40. Novick, R. P. et al. Synthesis of staphylococcal virulence factors is controlled by a regulatory RNA molecule. EMBO J. 12, 3967-3975 (1993).

41. Yoong, P. \& Torres, V. J. Counter inhibition between leukotoxins attenuates Staphylococcus aureus virulence. Nat. Commun. 6, 8125 (2015).

42. Kuehn, M. J. \& Kesty, N. C. Bacterial outer membrane vesicles and the hostpathogen interaction. Genes Dev. 19, 2645-2655 (2005).

43. Schertzer, J. W. \& Whiteley, M. Bacterial outer membrane vesicles in trafficking, communication and the host-pathogen interaction. J. Mol. Microbiol. Biotechnol. 23, 118-130 (2013).

44. Hwang, I. Cell-cell communication via extracellular membrane vesicles and its role in the immune response. Mol. Cells 36, 105-111 (2013).

45. Koeppen, K. et al. A novel mechanism of host-pathogen interaction through sRNA in bacterial outer membrane vesicles. PLoS Pathog. 12, e1005672 (2016).

46. Shen, Y. et al. Outer membrane vesicles of a human commensal mediate immune regulation and disease protection. Cell Host. Microbe 12, 509-520 (2012).

47. Gurung, M. et al. Staphylococcus aureus produces membrane-derived vesicles that induce host cell death. PLoS ONE 6, e27958 (2011).

48. Lee, E. Y. et al. Gram-positive bacteria produce membrane vesicles: proteomics-based characterization of Staphylococcus aureus-derived membrane vesicles. Proteomics 9, 5425-5436 (2009).

49. Jeon, H. et al. Variation among Staphylococcus aureus membrane vesicle proteomes affects cytotoxicity of host cells. Microb. Pathog. 93, 185-193 (2016).

50. Chatterjee, S. S. et al. Essential Staphylococcus aureus toxin export system. Nat. Med 19, 364-367 (2013).

51. Nazari, M., Kurdi, M. \& Heerklotz, H. Classifying surfactants with respect to their effect on lipid membrane order. Biophys. J. 102, 498-506 (2012).

52. Drin, G. \& Antonny, B. Amphipathic helices and membrane curvature. FEBS Lett. 584, 1840-1847 (2010).

53. Schlag, M. et al. Role of staphylococcal wall teichoic acid in targeting the major autolysin Atl. Mol. Microbiol. 75, 864-873 (2010).

54. Frankel, M. B. \& Schneewind, O. Determinants of murein hydrolase targeting to cross-wall of Staphylococcus aureus peptidoglycan. J. Biol. Chem. 287, 10460-10471 (2012)

55. Biswas, R. et al. Proton-binding capacity of Staphylococcus aureus wall teichoic acid and its role in controlling autolysin activity. PLOS ONE 7, e41415 (2012).

56. Surve, M. V. et al. Membrane vesicles of Group B Streptococcus disrupt fetomaternal barrier leading to preterm birth. PLoS Pathog. 12, e1005816 (2016).

57. Monteiro, J. M. et al. Cell shape dynamics during the staphylococcal cell cycle. Nat. Commun. 6, 8055 (2015)

58. Yamada, S. et al. An autolysin ring associated with cell separation of Staphylococcus aureus. J. Bacteriol. 178, 1565-1571 (1996).

59. Liu, B., Park, S., Thompson, C. D., Li, X. \& Lee, J. C. Antibodies to Staphylococcus aureus capsular polysaccharides 5 and 8 perform similarly in vitro but are functionally distinct in vivo. Virulence 8, 859-874 (2017).

60. Rodrigues, M. L. et al. Vesicular polysaccharide export in Cryptococcus neoformans is a eukaryotic solution to the problem of fungal trans-cell wall transport. Eukaryot. Cell 6, 48-59 (2007).

61. Bubeck Wardenburg, J. \& Schneewind, O. Vaccine protection against Staphylococcus aureus pneumonia. J. Exp. Med. 205, 287-294 (2008).

62. Rauch, S. et al. Abscess formation and alpha-hemolysin induced toxicity in a mouse model of Staphylococcus aureus peritoneal infection. Infect. Immun. 80, 3721-3732 (2012).

63. Kennedy, A. D. et al. Targeting of alpha-hemolysin by active or passive immunization decreases severity of USA300 skin infection in a mouse model. J. Infect. Dis. 202, 1050-1058 (2010).

64. Brown, L. E. \& Jackson, D. C. Lipid-based self-adjuvanting vaccines. Curr. Drug Deliv. 2, 383-393 (2005).

65. Basto, A. P. \& Leitao, A. Targeting TLR2 for vaccine development. J. Immunol Res. 2014, 619410 (2014)

66. Augustin, J. et al. Genetic analysis of epidermin biosynthetic genes and epidermin-negative mutants of Staphylococcus epidermidis. Eur. J. Biochem. 204, 1149-1154 (1992).

67. Bubeck Wardenburg, J., Williams, W. A. \& Missiakas, D. Host defenses against Staphylococcus aureus infection require recognition of bacterial lipoproteins. Proc. Natl Acad. Sci. USA 103, 13831-13836 (2006).

68. Wacker, M. et al. Prevention of Staphylococcus aureus infections by glycoprotein vaccines synthesized in Escherichia coli. J. Infect. Dis. 209, 1551-1561 (2014) 


\section{Acknowledgements}

We are grateful to Drs. Michael Otto for providing the S. aureus psm mutants, Jianxun Ding for providing assistance with DLS and nanotracking particle analysis experiments, and Matthew Waldor for use of the StepOnePlus Real-Time PCR System.

\section{Author contributions}

X.W. initiated the project, and X.W., C.W., and J.C.L designed experiments. X.W. performed the experiments with the assistance of C.D.T. All authors analyzed the data, and X.W., C.W., and J.C.L. wrote the manuscript. All authors reviewed the manuscript.

\section{Additional information}

Supplementary Information accompanies this paper at https://doi.org/10.1038/s41467018-03847-z.

Competing interests: The authors declare no competing interests.

Reprints and permission information is available online at http://npg.nature.com/ reprintsandpermissions/
Publisher's note: Springer Nature remains neutral with regard to jurisdictional claims in published maps and institutional affiliations.

(c) (i) Open Access This article is licensed under a Creative Commons Attribution 4.0 International License, which permits use, sharing, adaptation, distribution and reproduction in any medium or format, as long as you give appropriate credit to the original author(s) and the source, provide a link to the Creative Commons license, and indicate if changes were made. The images or other third party material in this article are included in the article's Creative Commons license, unless indicated otherwise in a credit line to the material. If material is not included in the article's Creative Commons license and your intended use is not permitted by statutory regulation or exceeds the permitted use, you will need to obtain permission directly from the copyright holder. To view a copy of this license, visit http://creativecommons.org/ licenses/by/4.0/.

(C) The Author(s) 2018 